Preprint no.

\title{
Neutrino emissivities and bulk viscosity in neutral two-flavor quark matter
}

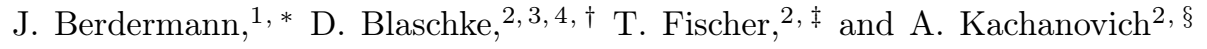 \\ ${ }^{1}$ Deutsches Zentrum für Luft- und Raumfahrt (DLR), DE-17235 Neustrelitz, Germany \\ ${ }^{2}$ Institute of Theoretical Physics, Uniwersity of Wroclaw, 50-204 Wroclaw, Poland \\ ${ }^{3}$ Bogoliubov Laboratory of Theoretical Physics, Joint Institute for Nuclear Research, 141980 Dubna, Russia \\ ${ }^{4}$ National Research Nuclear University (MEPhI), 115409 Moscow, Russia
}

\begin{abstract}
We study thermodynamic and transport properties for the isotropic color-spin-locking (iso-CSL) phase of two-flavor superconducting quark matter under compact star constraints within a NJL-type chiral quark model. Chiral symmetry breaking and the phase transition to superconducting quark matter leads to a density dependent change of quark masses, chemical potentials and diquark gap. A self-consistent treatment of these physical quantities influences on the microscopic calculations of transport properties. We present results for the iso-CSL direct URCA emissivities and bulk viscosities, which fulfill the constraints on quark matter derived from cooling and rotational evolution of compact stars. We compare our results with the phenomenologically successful, but yet heuristic $2 \mathrm{SC}+\mathrm{X}$ phase. We show that the microscopically founded iso-CSL phase can replace the purely phenomenological $2 \mathrm{SC}+\mathrm{X}$ phase in modern simulations of the cooling evolution for compact stars with color superconducting quark matter interior.
\end{abstract}

PACS numbers: 12.38.Mh, 24.85.+p, 26.60.+c, 97.60.-s

\section{INTRODUCTION}

Present astrophysical observational programmes monitoring compact stars (CS) have provided new, highquality data for their static properties, thermal and spin evolution. These modern measurements constrain the equation of state $(\mathrm{EoS})$ and the transport properties of dense matter in CS interiors [1], for a recent review see [2]. In particular, the evidence for high masses [35] and large radii [6] of CSs, suggests that the EoS at high densities must be sufficiently stiff. This prompts the question for the possibility of deconfined quark matter at CSs interiors [7]. In this debate, it has been demonstrated that microscopic models of quark matter EoS allow for extended quark cores of CS, while satisfying current mass and radius constraints [8]11. This offers still a broad spectrum of possible realizations of hybrid stars in nature, as classified recently in Ref. [12]. The two extreme scenarios are the masquerade case [13, where the corresponding quark-hadron hybrid stars appear to have almost identical static properties to pure neutron stars, and the high-mass twin case [14, 15] associated with a strong first-order phase transition. The latter can be identified by observing CSs with similar high masses (such as PSR J1614-2230 [3, 4] and PSR J0348+0432 [5]) but significantly different radii. This requires an accuracy of radius measuments of about $500 \mathrm{~m}$, as it shall be provided by the NICER mission of NASA [16], planned for launch in the near future. In the case of a smooth cross-over transition, i.e. the masquerade case, precise observations of CS

*Electronic address: jens.berdermann@dlr.de

${ }^{\dagger}$ Electronic address: david.blaschke@ift.uni.wroc.pl

‡Electronic address: tobias.fischer@ift.uni.wroc.pl

$\S$ Electronic address: akachanovich@ift.uni.wroc.pl mass and/or radii will not allow to provide evidence for the existence of quark matter at their interiors. In such a situation, the transport properties of dense matter may provide the decisive diagnostic tool via the cooling history of CS.

Besides cold CSs, also in protoneutron stars (PNS) the transport properties play a crucial role. PNSs are born hot and lepton rich in the violent event of a core-collapse supernova. They deleptonize and cool on a timescale on the order of 10-30 s via the emission of neutrinos of all flavors [17-22]. The appearance and role of quark matter in PNS and core-collapse supernovae has long been studied by means of conducting numerical studies [23 26], also in particular as the trigger of the actual supernova explosion via a strong first-order phase transition at high density. This launches a strong hydrodynamics shock wave, in addition of the standard supernova standing bounce shock, and the release of an outburst of neutrinos of all flavors [27]. Those neutrinos are released during the shock passage across the neutrinospheres of last scattering, located always at low densities where matter is composed of hadronic degrees of freedom. The future observation of such neutrino signal may reveal yet unknown details of the associated with the quark-hadron phase transition. The caveat in all these studies was the treatment of neutrino interactions in quark matter, which was treated at the level of nucleons only. This approximation is valid when temperatures are on the order of $10 \mathrm{MeV}$ or above. However, during the long-term evolution of deleptonizing protoneutron star, as the core temperature decreases below about $1 \mathrm{MeV}$, weak interactions at the quark level become important. Unlike in studies of cooling CS, where neutrino-quark interactions are treated at different levels of sophistication [28 30, for supernova studies the general framework has to be derived along the lines of Refs. 31, 32.

Since the cooling and spin evolution of CS depends sen- 
sitively on the thermal and transport properties of dense matter, the latter can be determined from the observation of cooling CSs, with particular emphasis on young objects like Cassiopeia A [33. For a recent discussion of the role of the stiffness of the EoS and the superfluidity gaps in this context, cf. Refs. [34, 35]. If quark matter is present in the CS interior we expect it to be in a color-superconducting state which entails a strong dependence on the pairing pattern and the sizes of pairing gaps. In the present study, we will focus on the discussion of direct Urca neutrino emissivities and bulk viscosities of color-superconducting quark matter. The numerical analysis is based on a Nambu-Jona-Lasinio (NJL) type model, allowing a consistent description of the density and temperature dependent quark masses, pairing gaps and chemical potentials under neutron star constraints. The resulting phase diagram suggests that three-flavor phases of the color-flavor-locking (CFL) type occur only at rather high densities [36, 37] and render hybrid star configurations gravitationally unstable [9, 38]. Moreover, due to large pairing gaps in CFL quark matter, the rmode instabilities cannot be damped [39] and cooling is inhibited [0].

By this reasoning, we shall focus on two-flavor quark matter as the relevant case for discussion of quark deconfinement in CSs as well as in the protoneutron star evolution during supernova collapse. Due to the pairing instability the scalar antitriplet diquark correlations form a condensate in the color superconducting $2 \mathrm{SC}$ phase with a critical temperature $T_{2 \mathrm{SC}}$ that is on the order of $20-50 \mathrm{MeV}$ [36, 37. Within the Polyakov-loop extension of the NJL model, this temperature may even reach up to the pseudocritical temperature $T_{c}=154 \mathrm{MeV}$ found in recent lattics QCD simulations [41, 42] for the chiral and Polyakov-loop transition at vanishing baryon number densities, see [43, 44].

The standard 2SC phase, however, pairs only two of the three colors (e.g., red-green) of quarks, leaving color unpaired (blue quarks in this example) on which then the rapid direct Urca cooling process may proceed, too rapid in comparison with compact star phenomenology. This problem has prompted the introduction of a purely phenomenological gap (X-gap) for the quarks of the unpaired color [45]. For a recent investigation of such a fully gapped 2SC phase see [46, 47], which may be contrasted to the transport [48] and cooling properties [49] in the original $2 \mathrm{SC}$ phase. In this context also the anisotropic crystalline color superconductivity phases have been discussed, which have been reviewed in [50, 51].

It is an unsatisfactory situation to have no candidate for the microscopic pairing pattern that could justify the phenomenological $\mathrm{X}$-phase in the $2 \mathrm{SC}+\mathrm{X}$ model of the fully gapped $2 \mathrm{SC}$ phase. One alternative is provided by the isotropic color-spin-locking (iso-CSL) phase suggested in [52, 53] modifying earlier work on spin-1 color superconducting phases [54]. The iso-CSL phase is a single flavor pairing scheme and therefore rather inert against isospin asymmetry and strong magnetic fields, thus qualifying as a robust pairing pattern for compact star applications. Technically the description of the transport and cooling properties of this phase follows that of the family of spin-1 color superconductors which have been studied in detail in [55].

In the present work, we will focus on two-flavor colorsuperconducting phases in $\mathrm{CSs}$, the $2 \mathrm{SC}+\mathrm{X}$ phase of Ref. [45], for which a detailed investigation of the cooling phenomenology for hybrid stars has already been worked out [56, [57, and the iso-CSL phase [52, 53, for which a consistent microscopic calculation of the direct Urca emissivity and the bulk viscosity will be presented here for the first time [58]. This will form the basis of further phenomenological in astrophysics, with applications to supernovae and CSs.

\section{THERMODYNAMICS OF ISO-CSL AND 2SC PHASES}

One can introduce a general thermodynamical potential which is in mean field approximation

$$
\begin{aligned}
& \Omega\left(\mu_{B}, \mu_{Q}, \mu_{8}, T\right)=\frac{\bar{\sigma}_{u}^{2}+\bar{\sigma}_{d}^{2}}{8 G_{S}}+\frac{\Delta_{u}^{2}+\Delta_{d}^{2}}{8 G_{D}} \\
& -2 \int \frac{d^{3} p}{(2 \pi)^{3}} \sum_{i=1}^{12}\left[\frac{\lambda_{i}}{2}+T \ln \left(1+e^{-\lambda_{i} / T}\right)\right]+\Omega_{l}-\Omega_{0},
\end{aligned}
$$

where $\lambda_{i}$ are the excitation energies for the corresponding modes.

Here $\Omega_{e}=-\mu_{Q}^{4} / 12 \pi^{2}-\mu_{Q}^{2} T^{2} / 6-7 \pi^{2} T^{4} / 180$ denotes the thermodynamic potential of ultra-relativistic electrons, where $\mu_{Q}=-\mu_{e}$, and $\Omega_{0}$ is the divergent vacuum contribution which has to be subtracted to assure vanishing energy and pressure of the vacuum.

\section{A. iso-CSL phase}

The gap matrix of the iso-CSL phase [52, 53] is

$$
\hat{\Delta}=\Delta\left(\gamma_{3} \lambda_{2}+\gamma_{2} \lambda_{5}+\gamma_{1} \lambda_{7}\right),
$$

a scalar product of the three antisymmetric color matrices with the spin matrices $\left(\gamma_{3}, \gamma_{2}, \gamma_{1}\right)$ [52], whereas the pairing pattern for the $2 \mathrm{SC}$ phase is

$$
\hat{\Delta}=\Delta\left(i \gamma_{5} \tau_{2} \lambda_{2}\right),
$$

coupling two different flavor with each other.

Note that in the iso-CSL phase all modes have a gap in the corresponding excitation spectra

$$
\lambda_{1}^{2}=\left(\epsilon_{u, e f f}(p)-\mu_{u, e f f}(p)\right)^{2}+\Delta_{u, e f f}^{2}(p),
$$


with the effective values

$$
\begin{aligned}
\epsilon_{u, e f f} & =\sqrt{p^{2}+M_{u, e f f}^{2}(p),} \\
M_{u, e f f}(p) & =\frac{\mu_{u}}{\mu_{u, e f f}(p)} M_{u}(p), \\
\mu_{u, e f f}(p) & =\mu_{u} \sqrt{1+\Delta_{u}^{2} /\left(\mu_{u}\right)^{2}}, \\
\Delta_{u, e f f}^{2}(p) & =a_{u, 1} \Delta_{u}^{2} .
\end{aligned}
$$

and

$$
\lambda_{3,5}^{2}(p)=\left(\epsilon_{u}(p)-\mu_{u}\right)^{2}+a_{u,(3,5)}(p) \Delta_{u}^{2},
$$

with the momentum-dependent coefficients

$$
\begin{aligned}
a_{u, 1}(p)= & \frac{M_{u}^{2}(p)}{\mu_{u, e f f}^{2}(p)} \\
a_{u,(3,5)}(p)= & \frac{1}{2}\left[5-\frac{p^{2}}{\epsilon_{u}(p) \mu_{u}}\right. \\
& \pm \sqrt{\left.\left(1-\frac{p^{2}}{\epsilon(p) \mu_{u}}\right)^{2}+\frac{8 M_{u}^{2}(p)}{\epsilon_{u}^{2}(p)}\right]}
\end{aligned}
$$

where $\epsilon_{u}(p)=\sqrt{p^{2}+M_{u}^{2}(p)}$.

The excitation energies $\lambda_{7-12}$ are obtained by changing the flavor $(u \rightarrow d)$ and the even modes by exchange of $\mu \rightarrow-\mu$ in Eqs. 47.

\section{B. 2SC phase}

In case of the $2 \mathrm{SC}$ phase four out of the twelve eigenvalues $\lambda_{a}$ belong to the ungapped blue quarks and are determined easily via textbook methods [59] as $\lambda_{1 . .4}=\epsilon_{f}(p) \pm$ $\mu_{f b}$. Here the dispersion relation $\epsilon_{f}(p)=\sqrt{p^{2}+M_{f}^{2}(p)}$ contains the dynamical mass function $M_{f}(p)=m_{f}+\phi_{f}$ for the two quark flavors $f=u, d$.

We have introduced the chemical potentials for the quarks of unpaired color $\mu_{u b}=\mu_{B} / 3+2 \mu_{Q} / 3-2 \mu_{8} / 2$ and $\mu_{d b}=\mu_{u b}-\mu_{Q}$.

The other eight eigenvalues $\lambda_{5-12}$ belong to the red and green quarks which are paired in the 2SC state and have therefore an identical eigenvalue spectrum. It is thus sufficient to determine the four eigenvalues for the real and symmetric matrices of the red quarks

$\mathcal{M}_{s}=\left|\begin{array}{cccc}-\mu_{d, r}+M_{d} & p & 0 & -\Delta \\ p & -\mu_{d, r}-M_{d} & \Delta & 0 \\ 0 & \Delta & \mu_{u, r}+M_{u} & p \\ -\Delta & 0 & p & \mu_{u, r}-M_{u}\end{array}\right|$ with $s= \pm$ (similar to the one discussed in 37, 60 for the CFL phase). The eigenvalues of the matrix $\sqrt{8}$ can be found as the roots of the characteristic polynomial

$$
\lambda^{4}+a_{3} \lambda^{3}+a_{2} \lambda^{2}+a_{1} \lambda+a_{0}=0
$$

with the coefficients

$$
\begin{aligned}
& a_{0}=\Delta^{4}+2 \Delta^{2}\left(M_{d} M_{u}+\mu_{d, r} \mu_{u, r}+p^{2}\right) \\
&+\left[M_{d}^{2}-\left(\mu_{d, r}\right)^{2}+p^{2}\right]\left[M_{u}^{2}-\left(\mu_{u, r}\right)^{2}+p^{2}\right] \\
& a_{1}=- 2\left(M_{u}^{2} \mu_{d, r}+\Delta^{2}\left(\mu_{d, r}-\mu_{u, r}\right)-M_{d}^{2} \mu_{u, r}\right. \\
&\left.+\left(\mu_{d, r}-\mu_{u, r}\right)\left(\mu_{d, r} \mu_{u, r}+p^{2}\right)\right) \\
& a_{2}=- 2 \Delta^{2}-M_{d}^{2}-M_{u}^{2}+\left(\mu_{d, r}\right)^{2}-4 \mu_{d, r} \mu_{u, r} \\
&+\left(\mu_{u, r}\right)^{2}-2 p^{2} \\
& a_{3}=2\left(\mu_{d, r}-\mu_{u, r}\right) .
\end{aligned}
$$

The four roots of the quartic equation can be calculated by use of the real solution of the cubic equation 61 .

$$
u^{3}-a_{2} u^{2}+\left(a_{1} a_{3}-4 a_{0}\right) u-\left(a_{1}^{2}+a_{0} a_{3}^{2}-4 a_{0} a_{2}\right)=0 .
$$

The real roots of the quartic equation are then the root of the quadratic equation

$v^{2}+\left[\frac{a_{3}}{2} \mp\left(\frac{a_{3}^{2}}{4}+u_{1}-a_{2}\right)^{\frac{1}{2}}\right] v+\frac{u_{1}}{2} \mp\left[\left(\frac{u_{1}}{2}\right)^{2}-a_{0}\right]^{\frac{1}{2}}=0$.

\section{Comparison}

The global minima of $\Omega\left(\mu_{B}, \mu_{Q}, \mu_{8}, T\right)$ in the space of the order parameters corresponds to the thermodynamical equilibrium and is solution of the following gapequations

$$
\frac{\partial \Omega\left(T, \mu_{f}\right)}{\partial \bar{\sigma}_{f}}=\frac{\partial \Omega\left(T, \mu_{f}\right)}{\partial \Delta_{f}}=\frac{\partial \Omega(T, \mu)}{\partial \bar{\omega}}=\frac{\partial \Omega(T, \rho)}{\partial \bar{\rho}}=0 .
$$

We investigate the phase diagram for the isotropic isoCSL and for the 2SC phase by use of the $\mathrm{M}(\mathrm{p}=0)=380$ $\mathrm{MeV}$ parameter set of 62 .

The order parameter and the chemical potential of up,down-quarks and electrons are shown (Figure 1) in dependence of the quark chemical potential $\mu$ for temperature $T=0$. If the 2SC-phase is partial suppressed or breaks up completely, then the iso-CSL phase is realised and the phase diagram for quark matter has the form of Figure 2 . 

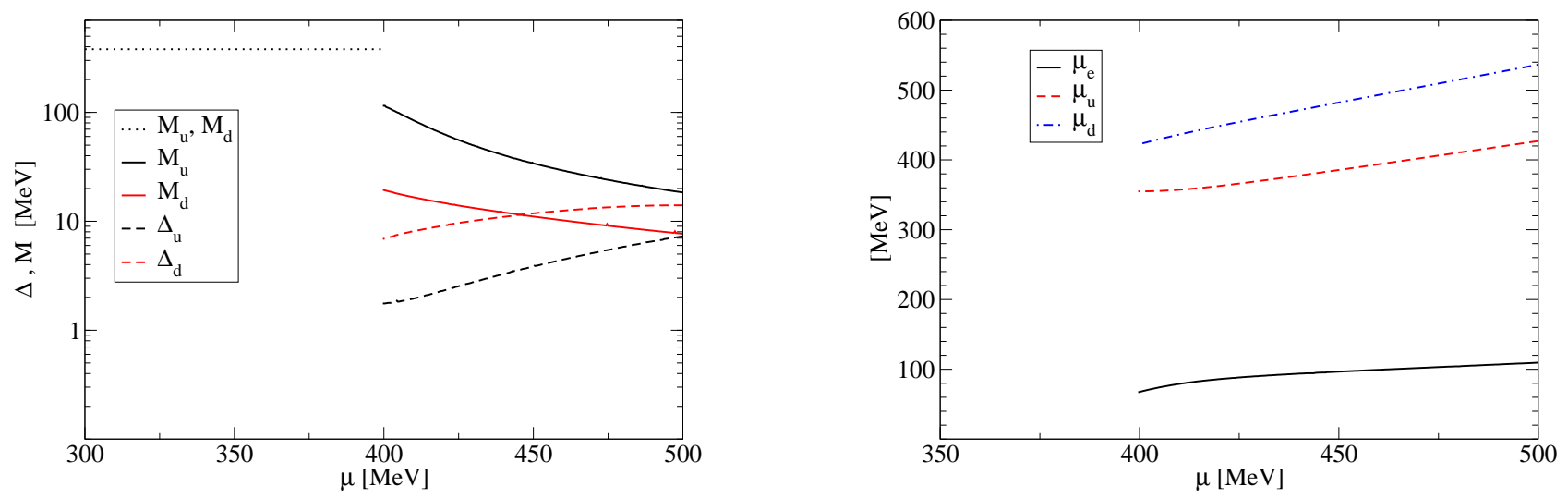

FIG. 1: The left panel shows the dynamical quark masses and pairing gaps $\left(\eta_{D}=3 / 8\right)$ for the iso-CSL phase as function of the quark-chemical potential and the corresponding chemical potentials for quarks and electrons are given in the right panel.
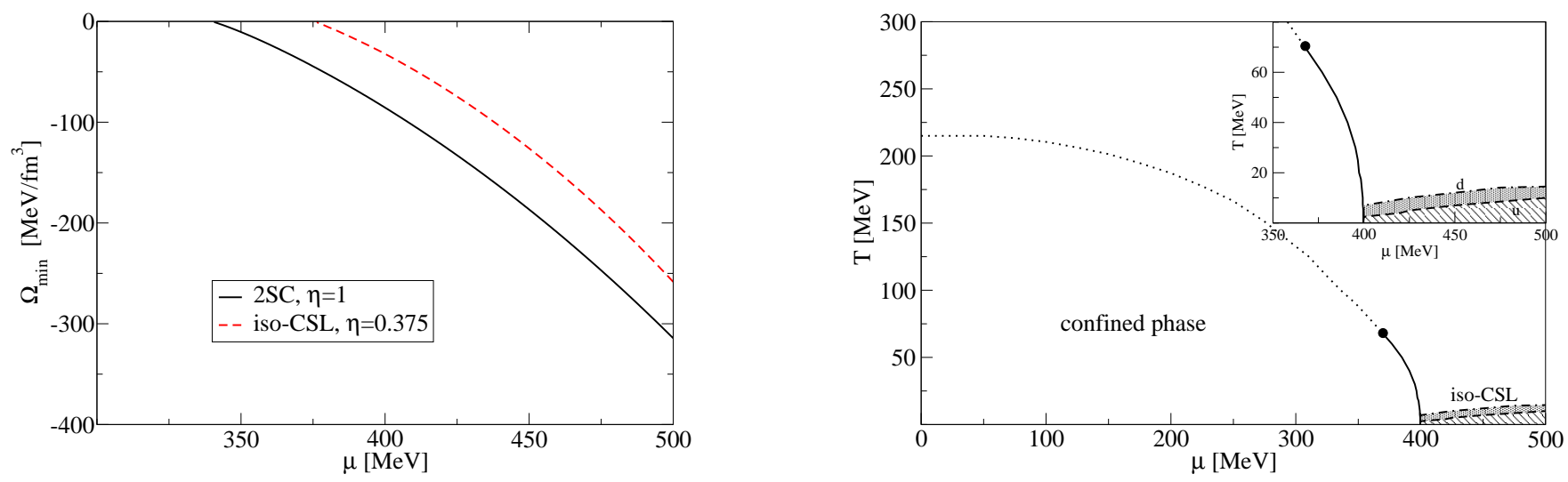

FIG. 2: The left panel displays the minima of the thermodynamical potential for 2SC and iso-CSL quark matter at $T=0$ as a function of the quark chemical potential. In the right panel the iso-CSL Phase diagram calculated with the NJL form factor and a diquark coupling of $\eta_{D}=3 / 8$ is shown.

\section{TRANSPORT PROPERTIES}

For the derivation of the kinetic equation for neutrinos in the iso-CSL and $2 \mathrm{SC}+\mathrm{X}$ phases of color superconducting quark matter and the corresponding direct Urca neutrino emissivities we follow the steps outlined in [55, 63, 64, while for the discussion of the bulk viscosity of these phases we adopt the formulation given in Ref. [65].

\section{A. Kinetic equation for neutrinos in warm dense quark matter}

The kinetic equation for neutrino transport in the Green's function representation,

$$
i \partial_{x}^{\alpha} \operatorname{Tr}_{\mathrm{D}}\left[\gamma_{\alpha} G_{\nu}^{<}\left(X, q_{2}\right)\right]=-\operatorname{Tr}\left[G_{\nu}^{>}\left(X, q_{2}\right) \Sigma_{\nu}^{<}\left(X, q_{2}\right)-\Sigma_{\nu}^{>}\left(X, q_{2}\right) G_{\nu}^{<}\left(X, q_{2}\right)\right]
$$


can be derived from the Kadanoff-Baym formalism [66] by a gradient expansion, which is valid if the neutrino Green's functions

$$
\begin{aligned}
& i G_{\nu}^{<}\left(t, q_{2}\right)=-\left(\gamma^{\beta} q_{2, \beta}+\mu_{\nu} \gamma_{0}\right) \frac{\pi}{q_{2}}\left\{f_{\nu}\left(t, \mathbf{q}_{\mathbf{2}}\right) \delta\left(p_{2}^{0}+\mu_{\nu}-\left|\mathbf{q}_{\mathbf{2}}\right|\right)-\left[1-f_{\bar{\nu}}\left(t,-\mathbf{q}_{\mathbf{2}}\right)\right] \delta\left(q_{2}^{0}+\mu_{\nu}+\left|\mathbf{q}_{\mathbf{2}}\right|\right)\right\} \\
& i G_{\nu}^{>}\left(t, q_{2}\right)=\left(\gamma^{\beta} q_{2, \beta}+\mu_{\nu} \gamma_{0}\right) \frac{\pi}{q_{2}}\left\{\left[1-f_{\nu}\left(t, \mathbf{q}_{\mathbf{2}}\right)\right] \delta\left(q_{2}^{0}+\mu_{\nu}-\left|\mathbf{q}_{\mathbf{2}}\right|\right)-f_{\bar{\nu}}\left(t,-\mathbf{q}_{\mathbf{2}}\right) \delta\left(q_{2}^{0}+\mu_{\nu}+\left|\mathbf{q}_{\mathbf{2}}\right|\right)\right\}
\end{aligned}
$$

and the neutrino self energies

$$
\begin{aligned}
& \Sigma_{\nu}^{<}\left(t, q_{2}\right)=\frac{G_{F}^{2}}{2} \int \frac{d^{4} q_{1}}{\left(2 \pi^{4}\right)} \gamma^{\mu}\left(1-\gamma_{5}\right)\left(\gamma^{\alpha} q_{1, \alpha}+\mu_{e} \gamma_{0}\right) \gamma^{\nu}\left(1-\gamma_{5}\right) \Pi_{\mu \nu}^{>}\left(q_{1}-q_{2}\right) \frac{\pi}{q_{1}} f_{e}\left(t, \mathbf{q}_{1}\right) \delta\left(q_{1}^{0}+\mu_{e}-\left|\mathbf{q}_{1}\right|\right), \\
& \Sigma_{\nu}^{>}\left(t, q_{2}\right)=\frac{G_{F}^{2}}{2} \int \frac{d^{4} q_{1}}{\left(2 \pi^{4}\right)} \gamma^{\mu}\left(1-\gamma_{5}\right)\left(\gamma^{\alpha} q_{1, \alpha}+\mu_{e} \gamma_{0}\right) \gamma^{\nu}\left(1-\gamma_{5}\right) \Pi_{\mu \nu}^{<}\left(q_{1}-q_{2}\right) \frac{\pi}{q_{1}}\left[1-f_{e}\left(t, \mathbf{q}_{1}\right)\right] \delta\left(q_{1}^{0}+\mu_{e}-\left|\mathbf{q}_{1}\right|\right)
\end{aligned}
$$

are slowly varying functions of the space-time coordinate $X=(t, \mathbf{x})$. The functions $\Pi_{\mu \nu}^{<,>}\left(q_{1}-q_{2}\right)$ are the self-energies of the W-bosons. The W-boson exchange can be expressed in its local form due to the smallness of the neutrinoenergy compared to the W-boson mass. We follow the steps of [55] to obtain the time dependent neutrino distribution function

$$
\frac{\partial}{\partial t} f_{\nu}\left(t, \mathbf{q}_{2}\right)=\frac{G_{F}^{2}}{8} \int \frac{d^{3} \mathbf{q}_{\mathbf{1}}}{(2 \pi)^{3} p_{F, e} p_{F, \nu}} \mathcal{L}^{\mu \nu}\left(q_{1}, q_{2}\right) n_{F}\left(p_{F, e}-\mu_{e}\right) n_{B}\left(p_{F, \nu}+\mu_{e}-p_{F, e}\right) \operatorname{Im}_{\mu \nu}^{R}(q)
$$

from Eq. 14, where

$$
\mathcal{L}^{\mu \nu}\left(q_{1}, q_{2}\right) \equiv \operatorname{Tr}\left[\left(\gamma_{0} q_{1}^{0}-\vec{\gamma} \cdot \mathbf{q}_{\mathbf{1}}\right) \gamma^{\mu}\left(1-\gamma^{5}\right)\left(\gamma_{0} q_{2}^{0}-\vec{\gamma} \cdot \mathbf{q}_{\mathbf{2}}\right) \gamma^{\nu}\left(1-\gamma^{5}\right)\right]
$$

is the leptonic tensor. We insert the imaginary part of the polarization tensor Eq. B11 and obtain

$$
\begin{aligned}
\frac{\partial}{\partial t} f_{\nu}\left(t, \mathbf{q}_{2}\right) & =-\frac{G_{F}^{2} \pi}{8} \cos ^{2} \theta_{c} \int \frac{d^{3} \mathbf{q}_{\mathbf{1}}}{(2 \pi)^{3} p_{F, e} p_{F, \nu}} \int \frac{d^{3} \mathbf{p}}{(2 \pi)^{3}} n_{F}\left(p_{F, e}-\mu_{e}\right) n_{B}\left(p_{F, \nu}+\mu_{e}-p_{F, e}\right) \\
& \times \sum_{r=1,3,5}\left[2 A_{r}^{*}\left(E_{p}, E_{k}\right) \mathcal{L}^{\mu \nu}\left(q_{1}, q_{2}\right) \mathcal{H}_{\mu \nu}^{(n)}(\hat{p}, \hat{k})-\Delta^{2} B_{r}^{*}\left(E_{p}, E_{k}\right) \mathcal{L}^{\mu \nu}\left(q_{1}, q_{2}\right) \mathcal{H}_{\mu \nu}^{(a)}(\hat{p}, \hat{k})\right] .
\end{aligned}
$$

To simplify this expression we can neglect the anomalous contribution $\mathcal{H}_{\mu \nu}^{a}$, which is small compared to the normal part $\mathcal{H}_{\mu \nu}^{n}[63$ and obtain

$$
\begin{aligned}
\frac{\partial}{\partial t} f_{\nu}\left(t, \mathbf{q}_{\mathbf{2}}\right) & =-\frac{G_{F}^{2} \pi}{4} \cos ^{2} \theta_{c} \int \frac{d^{3} \mathbf{q}_{\mathbf{1}}}{(2 \pi)^{3} p_{F, e} p_{F, \nu}} \int \frac{d^{3} \mathbf{p}}{(2 \pi)^{3}} n_{F}\left(p_{F, e}-\mu_{e}\right) \\
& \times \sum_{r=1,3,5} \sum_{e_{1}, e_{2}= \pm} B_{p}^{e_{1}} B_{k}^{e_{2}} n_{F}\left(-e_{1} \xi_{p, r}\right) n_{F}\left(e_{2} \xi_{k, r}\right) \delta\left(q_{0}+e_{1} \xi_{p, r}-e_{2} \xi_{k, r}\right) \mathcal{L}^{\mu \nu}\left(q_{1}, q_{2}\right) \mathcal{H}_{\mu \nu}^{(n)}(\hat{p}, \hat{k}),
\end{aligned}
$$

where the Bose functions cancel each other, because their argument represents the momentum transfer of the W-Boson $\left(p_{F, \nu}+\mu_{e}-p_{F, e}=-q_{0}=\xi_{p, r}-\xi_{k, r}\right)$. Contraction between leptonic and hadronic tensor leads to

$$
\mathcal{L}^{\mu \nu}\left(q_{1}, q_{2}\right) \mathcal{H}_{\mu \nu}^{(\mathrm{n})}(\hat{p}, \hat{k})=64 q_{1}^{0} q_{2}^{0}\left(1-\hat{q}_{1} \cdot \hat{p}\right)\left(1-\hat{q}_{2} \cdot \hat{k}\right)=64 q_{1}^{0} q_{2}^{0}\left(1-\cos \theta_{u e}\right)\left(1-\cos \theta_{\nu d}\right)
$$

In case of small angles $1-\cos \theta_{u e} \simeq \theta_{u e}^{2}$ and one obtains the simple expression

$$
\mathcal{L}^{\mu \nu}\left(q_{1}, q_{2}\right) \mathcal{H}_{\mu \nu}^{(\mathrm{n})}(\hat{p}, \hat{k})=64 q_{1}^{0} q_{2}^{0} \theta_{u e}^{2}\left(1-\cos \theta_{\nu d}\right)=64 p_{F, e} p_{F, \nu} \theta_{u e}^{2}\left(1-\cos \theta_{\nu d}\right)
$$

The angle $\theta_{u e}^{2}$ can be expressed by the angle $\theta_{d e}^{2}$, see Appendix C. Eq. C8 for the perturbative and the quark mass effect. 
We concentrate on the perturbative treatment, where $\theta_{d e}^{2}=(4 / 3 \pi) \alpha_{s}$ and the Boltzmann equation for the time evolution of the neutrino distribution function becomes

$$
\begin{aligned}
\frac{\partial}{\partial t} f_{\nu}\left(t, \mathbf{q}_{2}\right)= & -\frac{64}{3} \alpha_{s} G_{F}^{2} \cos ^{2} \theta_{c} \int \frac{d^{3} \mathbf{q}_{\mathbf{1}}}{(2 \pi)^{3}} \int \frac{d^{3} \mathbf{p}}{(2 \pi)^{3}} n_{F}\left(p_{F, e}-\mu_{e}\right) \sum_{r=1,3,5} \sum_{e_{1}, e_{2}= \pm} B_{p}^{e_{1}} B_{k}^{e_{2}} \\
& \times n_{F}\left(-e_{1} \xi_{p, r}\right) n_{F}\left(e_{2} \xi_{k, r}\right) \delta\left(p_{F, e}-\mu_{e}-p_{F, \nu}+e_{1} \xi_{p, r}-e_{2} \xi_{k, r}\right)\left(1-\cos \theta_{\nu d}\right) .
\end{aligned}
$$

Here the replacement $q_{0}=p_{F, e}-\mu_{e}-p_{F, \nu}$ has been made in the $\delta$-function of Eq. (20). The $\delta$-function of Eq. (23) vanishes if the angle between up- and down-quarks $\theta_{u d}$ corresponds to a fixed value $\theta_{0}$. The value of the angle $\theta_{0}$ ist given by $\cos \theta_{0} \equiv 1-\kappa \mu_{e}^{2} /\left(\mu_{u} \mu_{d}\right)$, with $\kappa \equiv 2 \alpha_{s} /(3 \pi)$. The angle $\theta_{0}$ is independent of the neutrino Fermi momentum $p_{F, \nu}$. The $\delta$-function can be replaced now by $\mu_{e} /\left(\mu_{u} \mu_{d}\right) \delta\left(\cos \theta_{u d}-\cos \theta_{0}\right)$ [55]. Eq. (23) becomes

$$
\begin{aligned}
\frac{\partial}{\partial t} f_{\nu}\left(t, \mathbf{q}_{2}\right) \simeq- & \frac{64}{3} \alpha_{s} G_{F}^{2} \cos ^{2} \theta_{c} \mu_{e} \mu_{u} \mu_{d} \int \frac{d k d \Omega_{k}}{(2 \pi)^{3}} \int \frac{d p d \Omega_{p}}{(2 \pi)^{3}}\left(1-\cos \theta_{\nu d}\right) \delta\left(\cos \theta_{u d}-\cos \theta_{0}\right) \\
& \times \sum_{r=1,3,5} \sum_{e_{1}, e_{2}= \pm} B_{p}^{e_{1}} B_{k}^{e_{2}} n_{F}\left(-e_{1} \xi_{p, r}\right) n_{F}\left(e_{2} \xi_{k, r}\right) n_{F}\left(p_{F, \nu}-\xi_{p, r}^{-}+\xi_{k, r}^{-}\right)
\end{aligned}
$$

where the variable of the integration $\mathbf{q}_{1}$ are changed to $\mathbf{k} \equiv \mathbf{p}+\mathbf{q}_{1}-\mathbf{q}_{2}$ and the phase space element for masseless quarks can be written as $d^{3} \mathbf{p}=\mu_{u}^{2} d p d \Omega_{p}$. The argument in the Fermi-distribution is replaced, because $p_{F, e}-\mu_{e}=$ $p_{F, \nu}-\xi_{p, r}^{-}+\xi_{k, r}^{-}$. Introducing dimensionless variables

$$
x \equiv \frac{k-\mu_{d}}{T}, \quad y \equiv \frac{p-\mu_{u}}{T}, \quad z \equiv \frac{q_{2}}{T} .
$$

changes the integration range $-\mu_{u, d} / T$ to $\infty$. As long as the main contribution results from $x, y \ll \mu_{u, d} / T$ the result of the integration is not affected by a shift of the lower boundary to $-\infty$. Therefore the uneven parts in the Bogoliubov coefficients

$$
\begin{aligned}
& B_{\mathbf{k}, r, d}^{e_{2}}=\frac{1}{2}-\frac{e_{2} x}{2 \sqrt{x^{2}+\lambda_{\mathbf{k}, r} \Delta_{d}^{2}}}, \\
& B_{\mathbf{p}, r, u}^{e_{2}}=\frac{1}{2}-\frac{e_{1} y}{2 \sqrt{y^{2}+\lambda_{\mathbf{p}, r} \Delta_{u}^{2}}},
\end{aligned}
$$

can be neglected in the integrand, thus only the even parts, a constant term $1 / 2$ remains [55]. The integral over $\mathrm{x}$ and $\mathrm{y}$ can now be done in the range from 0 to $\infty$, where one gets a factor 2 from the interval $-\infty \rightarrow 0$, which cancels with the constant term 1/2 from the Bogoliubov coefficients. Therefore, Eq. 24 becomes

$$
\frac{\partial}{\partial t} f_{\nu}\left(t, \mathbf{q}_{2}\right) \simeq-\frac{64}{3} \alpha_{s} G_{F}^{2} \cos ^{2} \theta_{c} \mu_{e} \mu_{u} \mu_{d} T^{2} \sum_{r=1,3,5} \int \frac{d \Omega_{k}}{(2 \pi)^{3}} \int \frac{d \Omega_{p}}{(2 \pi)^{3}}\left(1-\cos \theta_{\nu d}\right) \delta\left(\cos \theta_{u d}-\cos \theta_{0}\right) \mathcal{F}_{r}(z)
$$

with

$$
\mathcal{F}_{r}(z)=\sum_{e_{1}, e_{2}= \pm} \int_{0}^{\infty} \int_{0}^{\infty} d x d y\left(e^{-e_{1} \sqrt{y^{2}+a_{u, r} \Delta_{u}^{2}}}+1\right)^{-1}\left(e^{e_{2} \sqrt{x^{2}+a_{d, r} \Delta_{d}^{2}}}+1\right)^{-1}\left(e^{z+e_{1} \sqrt{y^{2}+a_{u, r} \Delta_{u}^{2}}-e_{2} \sqrt{x^{2}+a_{d, r} \Delta_{d}^{2}}}+1\right)^{-1} .
$$

\section{B. Emissivity}

The loss of energy by neutrinos per unit of time and volume is given by

$$
\varepsilon_{\nu} \equiv-\frac{\partial}{\partial t} \int \frac{\mathrm{d}^{3} \mathbf{q}_{\mathbf{2}}}{(2 \pi)^{3}}\left|\mathbf{q}_{\mathbf{2}}\right|\left[f_{\nu}\left(t, \mathbf{q}_{\mathbf{2}}\right)+f_{\bar{\nu}}\left(t, \mathbf{q}_{\mathbf{2}}\right)\right]=-2 \frac{\partial}{\partial t} \int \frac{\mathrm{d}^{3} \mathbf{q}_{\mathbf{2}}}{(2 \pi)^{3}} p_{F, \nu} f_{\nu}\left(t, \mathbf{q}_{\mathbf{2}}\right)
$$

The corresponding expression for the neutrino emissivity can be obtained by inserting Eq. 27. into Eq. 29. Performing the angle integration gives a factor $32 \pi^{3}$ [28], summation over the color states $r$ a factor 3 and by use of the integral

$$
\sum_{e_{1}, e_{2}= \pm} \int_{0}^{\infty} d z z^{3} \int_{0}^{\infty} d x \int_{0}^{\infty} d y\left(e^{-e_{1} y}+1\right)^{-1}\left(e^{e_{2} x}+1\right)^{-1}\left(e^{z+e_{1} y-e_{2} x}+1\right)^{-1}=\frac{457}{5040} \pi^{6}
$$

one obtains the gapless result of Iwamotos seminal paper [29] where the direct Urca emissivity of quark matter,

$$
\epsilon_{0}=\frac{457}{630} \alpha_{s} G_{F}^{2} \mu_{e} \mu_{u} \mu_{d} T^{6}
$$

was derived for the first time. Since then, there have been a number of calculations, in particular for color supercon- 
ducting phases, we refer to [55, 63, 64]. However, none of these is useable for cooling simulations because they have either ungapped modes which result in too fast cooling or the pairing pattern is not microscopically founded. Nevertheless, in deriving the neutrino emissivities for the $2 \mathrm{SC}+\mathrm{X}$ and the iso-CSL phase we follow the strategy of these References by using the form

$$
\epsilon_{\mathrm{Urca}}=\epsilon_{0} G_{3}\left(\Delta_{u}, \Delta_{d}\right)
$$

where we introduced the function

$G_{n}\left(\Delta_{u}, \Delta_{d}\right)=\frac{5040}{1371 \pi^{6}} \int_{0}^{\infty} d z z^{n}\left[\mathcal{F}_{1}(z)+\mathcal{F}_{3}(z)+\mathcal{F}_{5}(z)\right]$,

with Eq. 28 characterising the influence of the superconducting gaps on the corresponding emissivity. For the iso-CSL phase, the coefficients $a_{u, r}$ and $a_{d, r}$ for $r=1,3,5$ are defined in Ref. 52 and the gaps, obtained from the minimization of (1) fulfill in general $\Delta_{u} \neq \Delta_{d}$. In the $2 \mathrm{SC}+\mathrm{X}$ phase $\Delta_{u}=\Delta_{d}=\Delta$ and $a_{f, 1}=a_{f, 3}=1$, $a_{f, 5}=\left(\Delta_{X} / \Delta\right)^{2}$ for $f=u, d$. This simplifies Eq. 28) as long as no dependence of a density dependent strong coupling $\alpha_{s}$ is taken into account. In the CSL-phase the functions for the several modes and flavors are introduced in Eq. (7) with $p_{d}=x T+\mu_{d}, \quad p_{u}=y T+\mu_{u}$ and the dispersion relation $E_{f}=\sqrt{p_{f}^{2}+M_{f}^{2}}$. The formulas are presented in a way to allow comparison with the spin-1 phase from the work of [55] (see Fig. 3, lower panel). The density dependent X-gap $\Delta_{X}$ was introduced in Ref. [45] for the first time to appropriately fit the cooling data of CS. Here we use the parametrization denoted as model IV in Ref. [56], where $\Delta_{X}$ has been investigated more detailed to fulfill constraints from recent cooling phenomenology. The influence of the temperature dependence is taken into account by

$$
\Delta(T)=\Delta_{0} \sqrt{1-\left(T / T_{c}\right)^{\beta}},
$$

where one can find values for $\beta$ between $1.0-3.2$ in the literature. In the following calculations we use $\beta=1.0$.

In Figure 3 we show the emissivities for the microscopic iso-CSL phase (lower panel) in comparison with the purely phenomenological $2 \mathrm{SC}+\mathrm{X}$ phase (upper panel) as a function of temperature for different chemical potentials. For both phases a similar suppression of the emissivity is obtained. Hence the iso-CSL phase is probably able to explain recent cooling data in a more consistent way supporting the idea of superconducting phases in quark stars as explanation for observed fast CS cooling.

\section{Bulk viscosity}

According to 67, in the absence of viscosity all rotating CS would become unstable against r-modes 68.
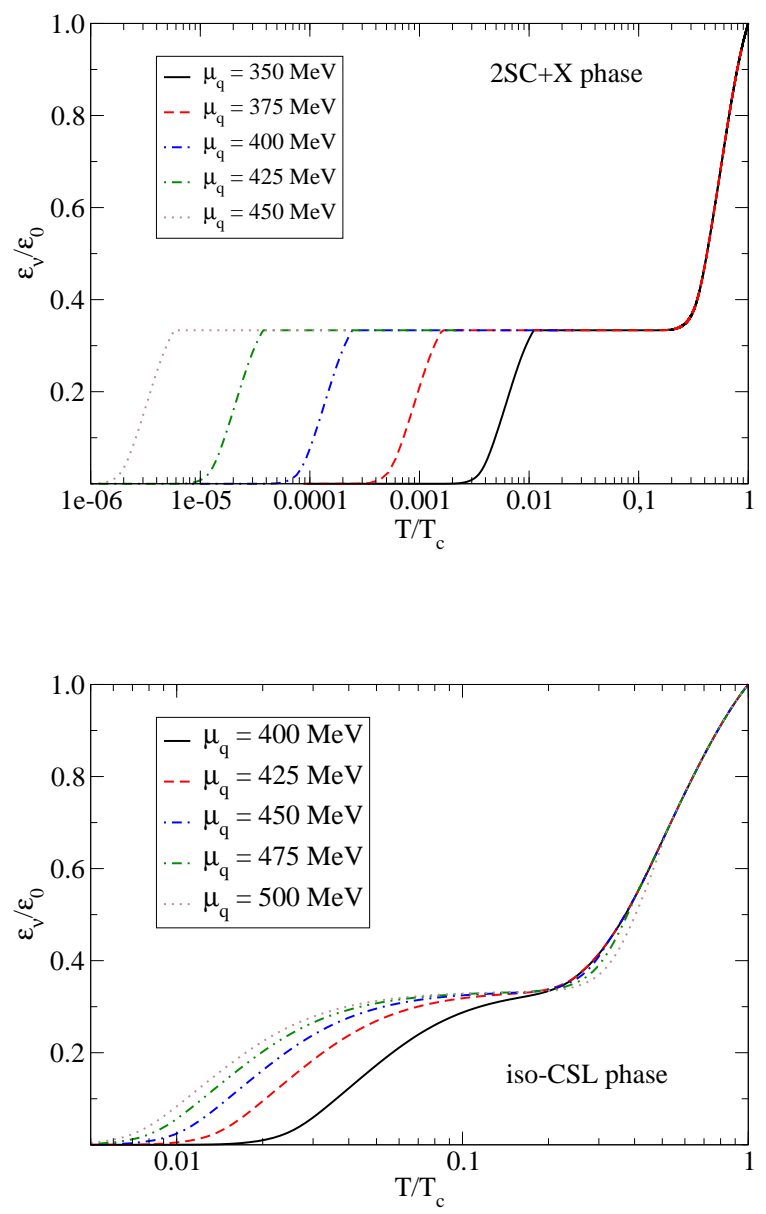

FIG. 3: Neutrino emissivities due to direct Urca processes in the $2 \mathrm{SC}+\mathrm{X}$ phase (upper panel) and in the iso-CSL phase (lower panel).

Therefore, from the observation of millisecond pulsars, one can derive constraints for the composition of CS interiors [39, 69]. For such an investigation, the bulk viscosity is a key quantity and we want to consider it for the two-flavor color superconducting phases introduced above, following the approach described in Ref. [65. Note that the 2SC phase considered in [70] is a threeflavor phase, where the nonleptonic process $u+d \leftrightarrow u+s$ provides the dominant contribution, see also [71. Due to absence of strange quarks in the $2 \mathrm{SC}$ phase of the present paper, this process does not occur.

The bulk viscosity at all temperatures is determined by 
with $C_{t}=C+C^{\prime}$ and the coefficients functions

$$
\begin{aligned}
C & =\frac{M_{u}^{2}}{3 \mu_{u}}-\frac{M_{d}^{2}}{3 \mu_{d}} \\
C^{\prime} & =\frac{4 \alpha_{s}}{3 \pi}\left[\frac{M_{d}^{2}}{\mu_{d}}\left(\ln \frac{2 \mu_{d}}{M_{d}}-\frac{2}{3}\right)-\frac{M_{u}^{2}}{\mu_{u}}\left(\ln \frac{2 \mu_{u}}{M_{u}}-\frac{2}{3}\right)\right] \\
B & \simeq \frac{\pi^{2}}{3} n\left(\frac{1}{\mu_{u}^{2}}+\frac{1}{\mu_{d}^{2}}+\frac{1}{\mu_{e}^{2}}\right) .
\end{aligned}
$$

The relevant processes for the bulk viscosity in twoflavor quark matter are the flavor changing weak processes of electron capture and beta decay, with a direct relation to the direct Urca emissivity

$$
\lambda=\frac{3}{2} \frac{\epsilon_{0}}{T^{2}} G_{1}\left(\Delta_{u}, \Delta_{d}\right) .
$$

The numerical results for the NJL model in selfconsistent meanfield approximation are displayed in Fig. 4 for the $2 \mathrm{SC}+\mathrm{X}$ phase (upper panel) and the isoCSL phase (lower panel) in striking similarity.

Note that in comparison with Ref. [65] the peak value of the viscosity is also located at $T=1 \sim 2 \mathrm{MeV}$, but up to three orders of magnitude higher! Since the normal quark matter results coincide, this must be a result of the self-consistent treatment of masses, gaps and composition (chemical potentials) in the present models. In particular the strongly density dependent X-gap is rapidly decreasing with increasing density as one can see by the dramatic change for the bulk viscosity at low quark chemical potentials.

\section{CONCLUSIONS}

Transport properties in dense quark matter depend sensitively on the color superconductivity pairing patterns and provide thus a tool for unmasking the CS interiors by their cooling and rotational evolution characteristics. On the example of neutrino emissivities and bulk viscosities for the $2 \mathrm{SC}+\mathrm{X}$ and the iso-CSL phase we have demonstrated that both two-flavor color superconducting phases fulfil constraints from the CS phenomenology. For the $2 \mathrm{SC}+\mathrm{X}$ phase with yet heuristic assumptions for the $\mathrm{X}$-gap the hybrid star configurations and their cooling evolution have been numerically evaluated in accordance with observational data. The temperature and density behavior of the neutrino emissivity in the microscopically well-founded iso-CSL phase appear rather similar so that we expect a good agreement with CS cooling data too. The bulk viscosities for both phases have been presented here for the first time and provide sufficient damping of r-mode instabilities to comply with the phenomenology of rapidly spinning CS. We conclude that the subtle interplay between suppression of the direct Urca cooling process on the one hand and sufficiently large bulk viscosity puts severe constraints on microscopic approaches to quark matter in compact stars.
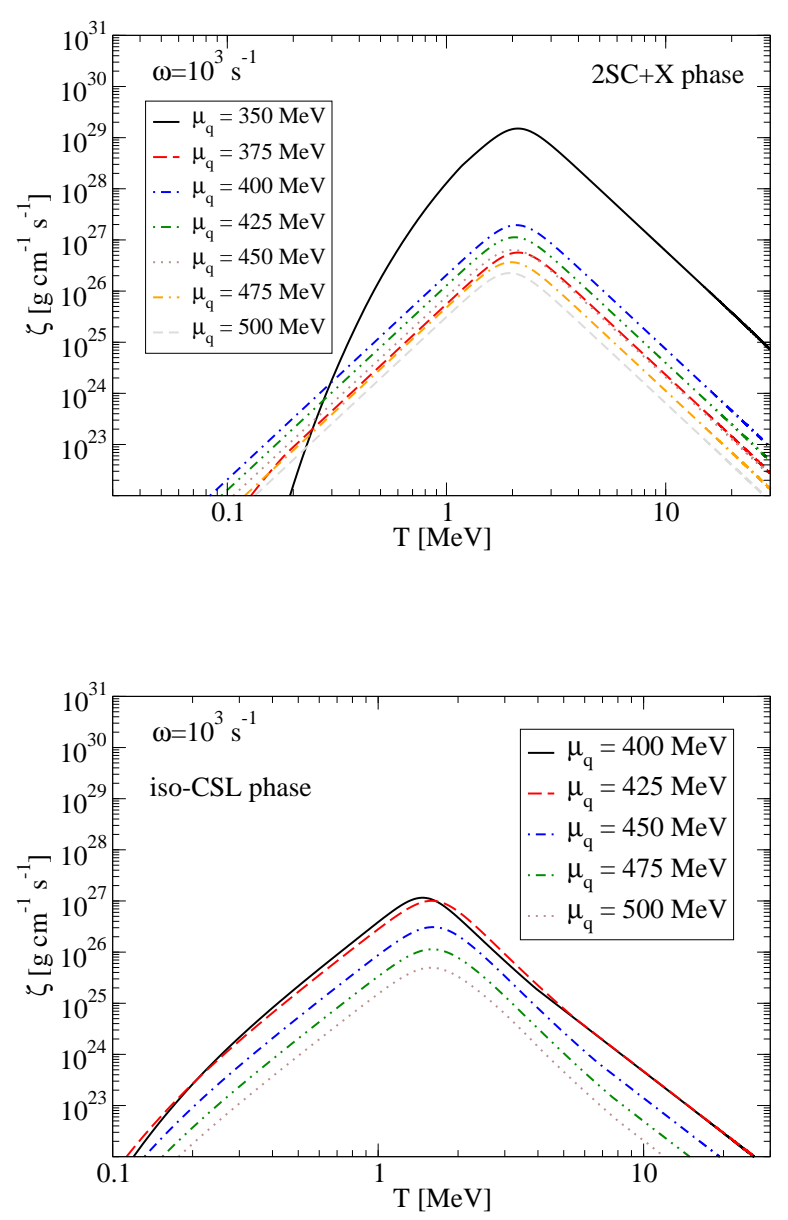

FIG. 4: Temperature dependence of bulk viscosity in the $2 \mathrm{SC}+\mathrm{X}$ phase (upper panel) and in the iso-CSL phase (lower panel) for a frequency of $\omega=1 \mathrm{kHz}$, typical for excitations of $\mathrm{r}$-modes in millisecond pulsars.

\section{ACKNOWLEDGEMENTS}

The authors acknowledge support from the Polish National Science Center (NCN) under grant numbers UMO-2011/02/A/ST2/00306 (A.K., D.B.) and UMO2014/15/B/ST2/03752 (T.F.). The work of D.B. was supported in part by the MEPhI Academic Excellence Project under contract number 02.a03.21.0005. 


\section{Appendix A: Quark propagator}

The free quark propagator $S_{0}(p)=(\not p-m)^{-1}$ can be represented as

$$
\begin{aligned}
S_{0}(p) & =\frac{1}{\not p-m}=\frac{\not p+m}{(\not p-m)(\not p+m)}=\frac{\not p+m}{p^{2}-m^{2}} \\
S_{0}^{ \pm}\left(p_{0}, \mathbf{p}\right) & =\frac{\not p+m}{\left(p_{0} \pm \mu\right)^{2}-\mathbf{p}^{2}-m^{2}}=\frac{\not p+m}{\left(p_{0} \pm \mu\right)^{2}-E_{p}^{2}} \\
& =\frac{\not p+m}{\left[p_{0} \pm \mu-E_{p}\right]\left[p_{0} \pm \mu+E_{p}\right]}
\end{aligned}
$$

with the particle energy $E_{p}=\sqrt{\mathbf{p}^{2}+m^{2}}$. The upper and lower sign corresponds to particle and antiparticle contribution. Eq. A1 can be extended to

$$
\begin{aligned}
S_{0}^{ \pm}\left(p_{0}, \mathbf{p}\right) & =\frac{\not p+m}{\left[p_{0} \pm \mu-E_{p}\right]\left[p_{0} \pm \mu+E_{p}\right]}, \\
& =\frac{1}{2 E_{p}}\left(\frac{\not p+m}{p_{0}-E_{p} \pm \mu}-\frac{\not p+m}{p_{0}+E_{p} \pm \mu}\right), \\
& =\frac{1}{2 E_{p}}\left(\frac{\gamma_{0}\left(p_{0} \pm \mu\right)-\gamma \mathbf{p}+m}{p_{0}-\left(E_{p} \mp \mu\right)}-\frac{\gamma_{0}\left(p_{0} \pm \mu\right)-\gamma \mathbf{p}+m}{p_{0}+\left(E_{p} \pm \mu\right)}\right), \\
& =\frac{1}{2 E_{p}}\left(\frac{\gamma_{0} E_{p}-\gamma \mathbf{p}+m}{p_{0}-E_{p}^{\mp}}+\frac{\gamma_{0} E_{p}+\gamma \mathbf{p}-m}{p_{0}+E_{p}^{ \pm}}\right),
\end{aligned}
$$

where the poles $p_{0, a}^{\mp}=+\left(E_{p} \mp \mu\right)$ and $p_{0, b}^{ \pm}=-\left(E_{p} \pm \mu\right)$ of the denominator have been replaced in the numerator. In addition we introduce the abbreviations $E_{p}^{\mp}=E_{p} \mp \mu$ and $E_{p}^{ \pm}=E_{p} \pm \mu$ in the denominator for the corresponding particle/hole- and antiparticle/anti-hole excitation energies. Simplifying one can write

$$
\begin{aligned}
S_{0}^{ \pm}\left(p_{0}, \mathbf{p}\right) & =\frac{1}{2 E_{p}}\left(\frac{\gamma_{0} E_{p}-\gamma \mathbf{p}+m}{p_{0}-E_{p}^{\mp}}+\frac{\gamma_{0} E_{p}+\gamma \mathbf{p}-m}{p_{0}+E_{p}^{ \pm}}\right) \\
& =\frac{\frac{1}{2}\left(\gamma_{0}-\frac{\gamma \mathbf{p}}{E_{p}}+\frac{m}{E_{p}}\right)}{p_{0}-E_{p}^{\mp}}+\frac{\frac{1}{2}\left(\gamma_{0}+\frac{\gamma \mathbf{p}}{E_{p}}-\frac{m}{E_{p}}\right)}{p_{0}+E_{p}^{ \pm}} \\
& =\frac{\gamma_{0} \frac{1}{2}\left(1-\gamma_{0} \gamma \hat{p}+\gamma_{0} \hat{m}\right)}{p_{0}-E_{p}^{\mp}}+\frac{\gamma_{0} \frac{1}{2}\left(1+\gamma_{0} \gamma \hat{p}-\gamma_{0} \hat{m}\right)}{p_{0}+E_{p}^{ \pm}} \\
& =\frac{\gamma_{0} \frac{1}{2}\left[1-\gamma_{0}(\gamma \hat{p}-\hat{m})\right]}{p_{0}-E_{p}^{\mp}}-\frac{\gamma_{0} \frac{1}{2}\left[1+\gamma_{0}(\gamma \hat{p}-\hat{m})\right]}{p_{0}+E_{p}^{ \pm}} \\
& =\frac{\gamma_{0} \tilde{\Lambda}_{p}^{-}}{p_{0}-E_{p}^{\mp}}+\frac{\gamma_{0} \tilde{\Lambda}_{p}^{+}}{p_{0}+E_{p}^{ \pm}},
\end{aligned}
$$

where the energy projectors are of the form $\Lambda_{p}^{ \pm}=\frac{1}{2}\left(1 \pm \gamma_{0} \mathcal{S}_{p}^{+}\right), \tilde{\Lambda}_{p}^{ \pm}=\frac{1}{2}\left(1 \pm \gamma_{0} \mathcal{S}_{p}^{-}\right)$and $\mathcal{S}_{p}^{ \pm}=\vec{\gamma} \hat{p} \pm \hat{m}$ are introduced with their corresponding "Foldy-Wouthhuysen" matrices and $\hat{m}=m / E_{p}$. The inverse free quark propagator is

$$
\left[S_{0}^{ \pm}\right]^{-1}=\gamma_{0}\left(p_{0}-E_{p}^{\mp}\right) \Lambda_{p}^{+}+\gamma_{0}\left(p_{0}+E_{p}^{ \pm}\right) \Lambda_{p}^{-} .
$$

To obtain the Nambu-Gorkov propagator we start with the identity

$$
\begin{aligned}
S^{-1} S & =\mathbf{1}, \\
\left(\begin{array}{cc}
{\left[S_{0}^{+}\right]^{-1}} & \Delta^{-} \\
\Delta^{+} & {\left[S_{0}^{-}\right]^{-1}}
\end{array}\right)\left(\begin{array}{cc}
A & B \\
C & D
\end{array}\right) & =\left(\begin{array}{ll}
1 & 0 \\
0 & 1
\end{array}\right),
\end{aligned}
$$

where we can find recursively the equations

$$
\begin{aligned}
& {\left[S_{0}^{+}\right]^{-1} A+\Delta^{-} C=1} \\
& {\left[S_{0}^{+}\right]^{-1} B+\Delta^{-} D=0} \\
& \Delta^{+} A+\left[S_{0}^{-}\right]^{-1} C=0 \\
& \Delta^{+} B+\left[S_{0}^{-}\right]^{-1} D=1
\end{aligned}
$$


from which we derive the implicit expressions

$$
\begin{aligned}
& A=\left[\left(S_{0}^{+}\right)^{-1}-\Sigma^{+}\right]^{-1}=G^{+} \\
& B=-S_{0}^{+} \Delta^{-} G^{-}=F^{-} \\
& C=-S_{0}^{-} \Delta^{+} G^{+}=F^{+} \\
& D=\left[\left(S_{0}^{-}\right)^{-1}-\Sigma^{-}\right]^{-1}=G^{-},
\end{aligned}
$$

for the normal and anomalous parts of the Nambu-Gorkov propagator with the selfenergies $\Sigma^{ \pm}=\Delta^{\mp} S_{0}^{\mp} \Delta^{ \pm}$where the gap matrix for two-flavor quark matter are $\Delta^{-}=-i \Delta \varepsilon^{i k} \epsilon^{\alpha \beta b} \gamma_{5}$ and $\Delta^{+}=-i \Delta^{*} \varepsilon^{i k} \epsilon^{\alpha \beta b} \gamma_{5}$. The Nambu-GorkovPropagator obtains the form

$$
S=\left(\begin{array}{cc}
G^{+} & F^{-} \\
F^{+} & G^{-}
\end{array}\right)
$$

with the normal parts

$$
G^{ \pm}=\left[\left(S_{0}^{ \pm}\right)^{-1}-\Delta^{\mp} S_{0}^{\mp} \Delta^{ \pm}\right]^{-1}=\frac{p_{0}+E_{p}^{\mp}}{p_{0}^{2}-\left(\xi_{p}^{\mp}\right)^{2}} \gamma_{0} \tilde{\Lambda}_{p}^{-}+\frac{p_{0}-E_{p}^{ \pm}}{p_{0}^{2}-\left(\xi_{p}^{ \pm}\right)^{2}} \gamma_{0} \tilde{\Lambda}_{p}^{+},
$$

and the anomalous parts

$$
F^{ \pm}=-S_{0}^{\mp} \Delta^{ \pm} G^{ \pm}=\frac{\Delta^{ \pm}}{p_{0}^{2}-\left(\xi_{p}^{ \pm}\right)^{2}} \tilde{\Lambda}_{p}^{+}+\frac{\Delta^{ \pm}}{p_{0}^{2}-\left(\xi_{p}^{\mp}\right)^{2}} \tilde{\Lambda}_{p}^{-}
$$

The four poles of the Nambu-Gorkov propagators $p_{0}= \pm \xi_{p}^{-}$and $p_{0}=\mp \xi_{p}^{+}$with $\left(\xi_{p}^{ \pm}\right)^{2}=\left(E_{p}^{ \pm}\right)^{2}+\Delta^{2}$ correspond to the quasi-particle/quasi-hole and quasi-antiparticle/quasi-anti-hole excitation energy in the color superconducting phase.

\section{Appendix B: Polarization tensor}

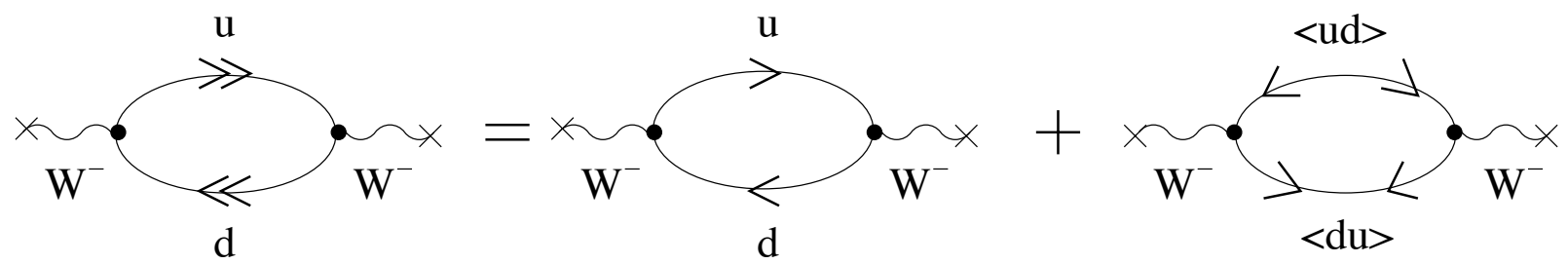

FIG. 5: Decomposition of the polarization loop diagram for the hadronic tensor into normal and anomalous parts.

The decomposition of the polarization loop diagram for the hadronic tensor in superconducting quark matter as shown in Fig. 5 has the following Nambu Gorkov structure

$$
\Pi_{\mu \nu}(q)=-\frac{i}{2} \int \frac{d^{4} p}{(2 \pi)^{4}} \operatorname{Tr}_{\mathrm{Z}, \mathrm{D}}\left[\Gamma_{\mu}^{Z} S_{p} \Gamma_{\nu}^{Z} S_{p+q}\right]
$$

with $\Gamma_{i}^{Z}=\left(\begin{array}{rc}\Gamma_{i}^{-} & 0 \\ 0 & \Gamma_{i}^{+}\end{array}\right)$, where $\Gamma_{i}^{ \pm}=\gamma_{\mu}\left(\mathcal{V} \pm \mathcal{A} \gamma_{5}\right)$ for $i=\mu, \nu$ and $S_{j}=\left(\begin{array}{cc}G_{j}^{+} & F_{j}^{-} \\ F_{j}^{+} & G_{j}^{-}\end{array}\right)$for $j=p, p+q$.

The vector and axial vector couplings of charged quark currents are $\mathcal{V}=\cos \theta_{c}$ and $\mathcal{A}=\cos \theta_{c}$ and the vertices have therefore the form $\Gamma_{i}^{ \pm}=\cos \theta_{c} \gamma_{\mu}\left(1 \pm \gamma_{5}\right)$. Performing the trace over the Nambu-Gorkov space leads to

$$
\Pi_{\mu \nu}(q)=-\frac{i}{2} \cos ^{2} \theta_{c} \int \frac{d^{4} p}{(2 \pi)^{4}} \operatorname{Tr}_{\mathrm{D}}\left[\Gamma_{\mu}^{-} G_{p}^{+} \Gamma_{\nu}^{-} G_{p+q}^{+}+\Gamma_{\mu}^{+} G_{p}^{-} \Gamma_{\nu}^{+} G_{p+q}^{-}+\Gamma_{\mu}^{-} F_{p}^{-} \Gamma_{\nu}^{+} F_{p+q}^{+}+\Gamma_{\mu}^{+} F_{p}^{+} \Gamma_{\nu}^{-} F_{p+q}^{-}\right]
$$

which can be expressed by

$$
\Pi_{\mu \nu}\left(q_{0}, \mathbf{q}\right)=-i \frac{T}{2} \cos ^{2} \theta_{c} \sum_{n} \int \frac{d^{3} \mathbf{p}}{(2 \pi)^{3}} \operatorname{Tr}_{\mathrm{D}}\left[\Gamma_{\mu}^{-} G_{p}^{+} \Gamma_{\nu}^{-} G_{p+q}^{+}+\Gamma_{\mu}^{+} G_{p}^{-} \Gamma_{\nu}^{+} G_{p+q}^{-}+\Gamma_{\mu}^{-} F_{p}^{-} \Gamma_{\nu}^{+} F_{p+q}^{+}+\Gamma_{\mu}^{+} F_{p}^{+} \Gamma_{\nu}^{-} F_{p+q}^{-}\right]
$$


as summation over fermionic $\left(p_{0}=i(2 n+1) \pi T\right)$ and bosonic $\left(q_{0}=i 2 m \pi T\right)$ Matsubara frequencies. The trace over the Dirac space indices results in

$$
\begin{aligned}
& \operatorname{Tr}_{\mathrm{D}}\left[\Gamma_{\mu}^{-} G_{p}^{+} \Gamma_{\nu}^{-} G_{p+q}^{+}+\Gamma_{\mu}^{+} G_{p}^{-} \Gamma_{\nu}^{+} G_{p+q}^{-}+\Gamma_{\mu}^{-} F_{p}^{-} \Gamma_{\nu}^{+} F_{p+q}^{+}+\Gamma_{\mu}^{+} F_{p}^{+} \Gamma_{\nu}^{-} F_{p+q}^{-}\right]= \\
= & \sum_{r}\left(\frac{\left(p_{0}+E_{p}^{-}\right)\left(p_{0}+q_{0}+E_{k}^{-}\right)}{\left[p_{0}^{2}-\xi_{p, r}^{2}\right]\left[\left(p_{0}+q_{0}\right)^{2}-\xi_{k, r}^{2}\right]}\left\{\mathcal{T}_{\mu \nu}^{+}(\hat{p}, \hat{k})+\widetilde{\mathcal{T}}_{\mu \nu}^{+}(\hat{p}, \hat{k})-\left[\widetilde{\mathcal{W}}_{\mu \nu}^{+}(\hat{p}, \hat{k})+\mathcal{W}_{\mu \nu}^{+}(\hat{p}, \hat{k})\right]\right\}\right. \\
+ & \frac{\left(p_{0}-E_{p}^{-}\right)\left(p_{0}+q_{0}-E_{k}^{-}\right)}{\left[p_{0}^{2}-\xi_{p, r}^{2}\right]\left[\left(p_{0}+q_{0}\right)^{2}-\xi_{k, r}^{2}\right]}\left\{\mathcal{T}_{\mu \nu}^{-}(\hat{p}, \hat{k})+\widetilde{\mathcal{T}}_{\mu \nu}^{-}(\hat{p}, \hat{k})+\left[\widetilde{\mathcal{W}}_{\mu \nu}^{-}(\hat{p}, \hat{k})+\mathcal{W}_{\mu \nu}^{-}(\hat{p}, \hat{k})\right]\right\} \\
- & \frac{\Delta^{2}}{\left[p_{0}^{2}-\xi_{p, r}^{2}\right]\left[\left(p_{0}+q_{0}\right)^{2}-\xi_{k, r}^{2}\right]}\left\{\mathcal{T}_{\mu \nu}^{-}(\hat{p}, \hat{k})+\mathcal{T}_{\mu \nu}^{+}(\hat{p}, \hat{k})+\widetilde{\mathcal{T}}_{\mu \nu}^{-}(\hat{p}, \hat{k})+\widetilde{\mathcal{T}}_{\mu \nu}^{+}(\hat{p}, \hat{k})\right. \\
- & {\left.\left.\left[\widetilde{\mathcal{W}}_{\mu \nu}^{+}(\hat{p}, \hat{k})+\mathcal{W}_{\mu \nu}^{+}(\hat{p}, \hat{k})-\widetilde{\mathcal{W}}_{\mu \nu}^{-}(\hat{p}, \hat{k})-\mathcal{W}_{\mu \nu}^{-}(\hat{p}, \hat{k})\right]\right\}\right) }
\end{aligned}
$$

with the tensors $\mathcal{T}_{\mu \nu}^{ \pm}(\hat{p}, \hat{k})=\operatorname{Tr}\left[\gamma_{0} \gamma_{\mu} \Lambda_{p}^{ \pm} \gamma_{0} \gamma_{\nu} \Lambda_{k}^{ \pm}\right], \mathcal{W}_{\mu \nu}^{ \pm}(\hat{p}, \hat{k})=\operatorname{Tr}\left[\gamma_{0} \gamma_{\mu} \Lambda_{p}^{ \pm} \gamma_{0} \gamma_{\nu} \Lambda_{k}^{ \pm} \gamma_{5}\right], \tilde{\mathcal{T}}_{\mu \nu}^{ \pm}(\hat{p}, \hat{k})=\operatorname{Tr}\left[\gamma_{0} \gamma_{\mu} \widetilde{\Lambda}_{p}^{ \pm} \gamma_{0} \gamma_{\nu} \Lambda_{k}^{ \pm}\right]$ and $\widetilde{\mathcal{W}}_{\mu \nu}^{ \pm}(\hat{p}, \hat{k})=\operatorname{Tr}\left[\gamma_{0} \gamma_{\mu} \widetilde{\Lambda}_{p}^{ \pm} \gamma_{0} \gamma_{\nu} \Lambda_{k}^{ \pm} \gamma_{5}\right]$

We introduce the notation $k=p+q$ for simplification. Performing the Matsubara summation results in the expressions

$$
\begin{aligned}
A^{ \pm}\left(E_{p}, E_{k}\right) & =T \sum_{n} \frac{\left(p_{0} \pm E_{p}^{-}\right)\left(p_{0}+q_{0} \pm E_{k}^{-}\right)}{\left[p_{0}^{2}-\left(\xi_{p}^{-}\right)^{2}\right]\left[\left(p_{0}+q_{0}\right)^{2}-\left(\xi_{k}^{-}\right)^{2}\right]}=-\frac{1}{2 \xi_{p}^{-} 2 \xi_{k}^{-}} \sum_{s_{1} s_{2}= \pm} \frac{\left(\xi_{p}^{-}+s_{1} E_{p}^{-}\right)\left(\xi_{k}^{-}+s_{2} E_{k}^{-}\right) n_{F}\left( \pm s_{1} \xi_{p}^{-}\right) n_{F}\left(\mp s_{2} \xi_{k}^{-}\right)}{q_{0} \pm s_{1} \xi_{p}^{-} \mp s_{2} \xi_{k}^{-}} \\
B\left(E_{p}, E_{k}\right) & =T \sum_{n} \frac{\Delta^{2}\left( \pm s_{1} \xi_{p}^{-} \mp s_{2} \xi_{k}^{-}\right)}{\left[p_{0}^{2}-\left(\xi_{p}^{-}\right)^{2}\right]\left[\left(p_{0}+q_{0}\right)^{2}-\left(\xi_{k}^{-}\right)^{2}\right]}=-\frac{1}{2 \xi_{p}^{-} 2 \xi_{k}^{-}} \sum_{s_{1} s_{2}= \pm} \frac{1}{q_{0}+s_{1} \xi_{p}^{-}-s_{2} \xi_{k}^{-}} \frac{n_{F}\left(s_{1} \xi_{p}^{-}\right) n_{F}\left(-s_{2} \xi_{k}^{-}\right)}{n_{B}\left(s_{1} \xi_{p}^{-}-s_{2} \xi_{k}^{-}\right)}
\end{aligned}
$$

so that we obtain for the polarization loop

$$
\begin{aligned}
\Pi_{\mu \nu}\left(q_{0}, \mathbf{q}\right)=-i & \sum_{r} \int \frac{\mathrm{d}^{3} \mathbf{p}}{(2 \pi)^{3}} A_{r}^{+}\left(E_{p}, E_{k}\right)\left\{\mathcal{T}_{\mu \nu}^{+}(\hat{p}, \hat{k})+\widetilde{\mathcal{T}}_{\mu \nu}^{+}(\hat{p}, \hat{k})-\left[\widetilde{\mathcal{W}}_{\mu \nu}^{+}(\hat{p}, \hat{k})+\mathcal{W}_{\mu \nu}^{+}(\hat{p}, \hat{k})\right]\right\} \\
& +A_{r}^{-}\left(E_{p}, E_{k}\right)\left\{\mathcal{T}_{\mu \nu}^{-}(\hat{p}, \hat{k})+\widetilde{\mathcal{T}}_{\mu \nu}^{-}(\hat{p}, \hat{k})+\left[\widetilde{\mathcal{W}}_{\mu \nu}^{-}(\hat{p}, \hat{k})+\mathcal{W}_{\mu \nu}^{-}(\hat{p}, \hat{k})\right]\right\} \\
& -\Delta^{2} B_{r}\left(E_{p}, E_{k}\right)\left\{\mathcal{T}_{\mu \nu}^{-}(\hat{p}, \hat{k})+\mathcal{T}_{\mu \nu}^{+}(\hat{p}, \hat{k})+\widetilde{\mathcal{T}}_{\mu \nu}^{-}(\hat{p}, \hat{k})+\widetilde{\mathcal{T}}_{\mu \nu}^{+}(\hat{p}, \hat{k})\right. \\
& \left.-\left[\widetilde{\mathcal{W}}_{\mu \nu}^{+}(\hat{p}, \hat{k})+\mathcal{W}_{\mu \nu}^{+}(\hat{p}, \hat{k})-\widetilde{\mathcal{W}}_{\mu \nu}^{-}(\hat{p}, \hat{k})-\mathcal{W}_{\mu \nu}^{-}(\hat{p}, \hat{k})\right]\right\}
\end{aligned}
$$

with the corresponding abbreviations

$$
\begin{array}{r}
A_{r}^{ \pm}\left(E_{p}, E_{k}\right)=\left[\frac{B_{p}^{\mp} B_{k}^{\mp}}{q_{0}-\xi_{p, r}+\xi_{k, r}}-\frac{B_{p}^{ \pm} B_{k}^{ \pm}}{q_{0}+\xi_{p, r}-\xi_{k, r}}\right] \frac{n_{F}\left(\xi_{p, r}\right) n_{F}\left(-\xi_{k, r}\right)}{n_{B}\left(\xi_{p, r}-\xi_{k, r}\right)} \\
+\left[\frac{B_{p}^{\mp} B_{k}^{ \pm}}{q_{0}-\xi_{p, r}-\xi_{k, r}}-\frac{B_{p}^{ \pm} B_{k}^{\mp}}{q_{0}+\xi_{p, r}+\xi_{k, r}}\right] \frac{n_{F}\left(\xi_{p, r}\right) n_{F}\left(\xi_{k, r}\right)}{n_{B}\left(\xi_{p, r}+\xi_{k, r}\right)} \\
B_{r}\left(E_{p}, E_{k}\right)=\frac{1}{4 \xi_{p, r} \xi_{k, r}}\left[\frac{1}{q_{0}-\xi_{p, r}+\xi_{k, r}}-\frac{1}{q_{0}+\xi_{p, r}-\xi_{k, r}}\right] \frac{n_{F}\left(\xi_{p, r}\right) n_{F}\left(-\xi_{k, r}\right)}{n_{B}\left(\xi_{p, r}-\xi_{k, r}\right)} \\
+\left[\frac{1}{q_{0}+\xi_{p, r}+\xi_{k, r}}-\frac{1}{q_{0}-\xi_{p, r}-\xi_{k, r}}\right] \frac{n_{F}\left(\xi_{p, r}\right) n_{F}\left(\xi_{k, r}\right)}{n_{B}\left(\xi_{p, r}+\xi_{k, r}\right)} .
\end{array}
$$

and the Bogoliubov coefficients

$$
B_{i}^{e} \equiv \frac{\xi_{i, r}+e E_{i}^{-}}{2 \xi_{i, r}}, \quad(i=p, k ; \quad e= \pm)
$$


The first term and the second term on the right hand side of Eq. (B6) provide the same contribution, since the second term is the charge conjugated counterpart of the first one.

$$
\begin{aligned}
\Pi_{\mu \nu}\left(q_{0}, \mathbf{q}\right)= & -i \sum_{r} \int \frac{\mathrm{d}^{3} \mathbf{p}}{(2 \pi)^{3}}\left(2 A_{r}\left(E_{p}, E_{k}\right)\left\{\mathcal{T}_{\mu \nu}^{+}(\hat{p}, \hat{k})+\widetilde{\mathcal{T}}_{\mu \nu}^{+}(\hat{p}, \hat{k})-\left[\widetilde{\mathcal{W}}_{\mu \nu}^{+}(\hat{p}, \hat{k})+\mathcal{W}_{\mu \nu}^{+}(\hat{p}, \hat{k})\right]\right\}\right. \\
& -\Delta^{2} B_{r}\left(E_{p}, E_{k}\right)\left\{\mathcal{T}_{\mu \nu}^{-}(\hat{p}, \hat{k})+\mathcal{T}_{\mu \nu}^{+}(\hat{p}, \hat{k})+\widetilde{\mathcal{T}}_{\mu \nu}^{-}(\hat{p}, \hat{k})+\widetilde{\mathcal{T}}_{\mu \nu}^{+}(\hat{p}, \hat{k})\right. \\
& \left.\left.-\left[\widetilde{\mathcal{W}}_{\mu \nu}^{+}(\hat{p}, \hat{k})+\mathcal{W}_{\mu \nu}^{+}(\hat{p}, \hat{k})-\widetilde{\mathcal{W}}_{\mu \nu}^{-}(\hat{p}, \hat{k})-\mathcal{W}_{\mu \nu}^{-}(\hat{p}, \hat{k})\right]\right\}\right) .
\end{aligned}
$$

The hadronic tensors $\mathcal{W}_{\mu \nu}^{ \pm}(\hat{p}, \hat{k})$ and $\widetilde{\mathcal{W}}_{\mu \nu}^{ \pm}(\hat{p}, \hat{k})$ are identical (Appendix $\mathrm{C}$ and the expression for the polarization tensor becomes

$$
\begin{aligned}
\Pi_{\mu \nu}\left(q_{0}, \mathbf{q}\right)= & -i \sum_{r} \int \frac{\mathrm{d}^{3} \mathbf{p}}{(2 \pi)^{3}}\left(2 A_{r}\left(E_{p}, E_{k}\right)\left[\mathcal{T}_{\mu \nu}^{+}(\hat{p}, \hat{k})+\widetilde{\mathcal{T}}_{\mu \nu}^{+}(\hat{p}, \hat{k})-2 \mathcal{W}_{\mu \nu}^{+}(\hat{p}, \hat{k})\right]\right. \\
& \left.-\Delta^{2} B_{r}\left(E_{p}, E_{k}\right)\left\{\mathcal{T}_{\mu \nu}^{-}(\hat{p}, \hat{k})+\mathcal{T}_{\mu \nu}^{+}(\hat{p}, \hat{k})+\widetilde{\mathcal{T}}_{\mu \nu}^{-}(\hat{p}, \hat{k})+\widetilde{\mathcal{T}}_{\mu \nu}^{+}(\hat{p}, \hat{k})-2\left[\mathcal{W}_{\mu \nu}^{+}(\hat{p}, \hat{k})-\mathcal{W}_{\mu \nu}^{-}(\hat{p}, \hat{k})\right]\right\}\right)(
\end{aligned}
$$

By analytic expansion and use of the Dirac identity

$$
\frac{1}{(x+i \eta)^{n+1}}=\mathcal{P} \frac{1}{x^{n+1}}-i \pi \frac{(-1)^{n}}{n !} \delta^{(n)}(x)
$$

one can extract the imaginary part of the polarization tensor

$$
\operatorname{Im} \Pi_{\mu \nu}^{R}\left(q_{0}, \mathbf{q}\right)=-\pi \cos ^{2} \theta_{c} \sum_{r} \int \frac{\mathrm{d}^{3} \mathbf{p}}{(2 \pi)^{3}}\left(2 A_{r}^{*}\left(E_{p}, E_{k}\right) \mathcal{H}_{\mu \nu}^{(\mathrm{n})}(\hat{p}, \hat{k})-\Delta^{2} B_{r}^{*}\left(E_{p}, E_{k}\right) \mathcal{H}_{\mu \nu}^{(\mathrm{a})}(\hat{p}, \hat{k})\right)
$$

where

$$
\begin{aligned}
& A_{r}^{*}\left(E_{p}, E_{k}\right)=\sum_{e_{1}, e_{2}= \pm} B_{p}^{e_{1}} B_{k}^{e_{2}} \frac{n_{F}\left(-e_{1} \xi_{p, r}\right) n_{F}\left(e_{2} \xi_{k, r}\right)}{n_{B}\left(-e_{1} \xi_{p, r}+e_{2} \xi_{k, r}\right)} \delta\left(q_{0}+e_{1} \xi_{p, r}-e_{2} \xi_{k, r}\right) \\
& B_{r}^{*}\left(E_{p}, E_{k}\right)=\sum_{e_{1}, e_{2}= \pm} \frac{e_{1} e_{2}}{4 \xi_{p, r} \xi_{k, r}} \frac{n_{F}\left(e_{1} \xi_{p, r}\right) n_{F}\left(-e_{2} \xi_{k, r}\right)}{n_{B}\left(e_{1} \xi_{p, r}-e_{2} \xi_{k, r}\right)} \delta\left(q_{0}-e_{1} \xi_{p, r}+e_{2} \xi_{k, r}\right) .
\end{aligned}
$$

The normal and anomalous hadronic tensors are

$$
\begin{aligned}
& \mathcal{H}_{\mu \nu}^{(\mathrm{n})}=\mathcal{T}_{\mu \nu}^{+}(\hat{p}, \hat{k})+\widetilde{\mathcal{T}}_{\mu \nu}^{+}(\hat{p}, \hat{k})-2 \mathcal{W}_{\mu \nu}^{+}(\hat{p}, \hat{k}) \\
& \mathcal{H}_{\mu \nu}^{(\mathrm{a})}=\mathcal{T}_{\mu \nu}^{-}(\hat{p}, \hat{k})+\mathcal{T}_{\mu \nu}^{+}(\hat{p}, \hat{k})+\widetilde{\mathcal{T}}_{\mu \nu}^{-}(\hat{p}, \hat{k})+\widetilde{\mathcal{T}}_{\mu \nu}^{+}(\hat{p}, \hat{k})-2\left[\mathcal{W}_{\mu \nu}^{+}(\hat{p}, \hat{k})-\mathcal{W}_{\mu \nu}^{-}(\hat{p}, \hat{k})\right] .
\end{aligned}
$$

\section{Appendix C: Kinematics}

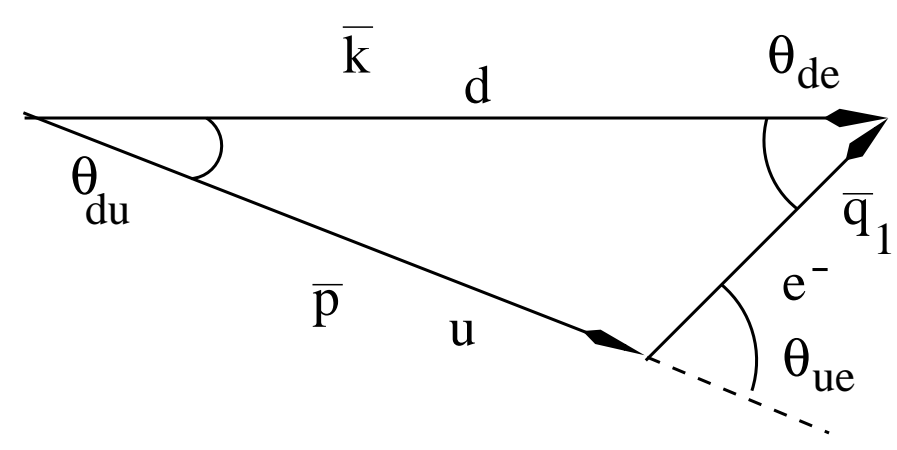

FIG. 6: Triangle of momentum conservation for the direct Urca process 
From Fig. 6 one finds the relations

$$
\begin{aligned}
p_{F, u} \cos \theta_{d u}+p_{F, e} \cos \theta_{d e} & =p_{F, d}, \\
p_{F, u} \sin \theta_{d u} & =p_{F, e} \sin \theta_{d e} .
\end{aligned}
$$

The cosine and sinus of the angles $\theta_{d u}$ and $\theta_{d e} \ll 1$ can be expanded in a power series to second order

$$
\begin{aligned}
p_{F, d}-p_{F, u}-p_{F, e} & =-\frac{1}{2}\left(p_{F, u} \theta_{d u}^{2}+p_{F, e} \theta_{d e}^{2}\right), \\
p_{F, u} \theta_{d u} & =p_{F, e} \theta_{d e} .
\end{aligned}
$$

Eliminating $\theta_{d u}$ by inserting $\mathrm{C} 4$ to $\mathrm{C} 3$ one gets in lowest order of $\theta_{d e}$ and $p_{F, e} / p_{F, u}$

$$
p_{F, d}-p_{F, u}-p_{F, e} \simeq-\frac{1}{2} p_{F, e} \theta_{d e}^{2}\left(1+\frac{p_{F, e}}{p_{F, u}}\right) \simeq-\frac{1}{2} p_{F, e} \theta_{d e}^{2}
$$

which corresponds to the momentum conservation of the direct Urca process. Note that $\theta_{d e} \simeq \theta_{u e}$. Momentum conservation ensures that all particles are collinear, as long as quark masses are neglected or no perturbative corrections in the dispersion relation of the free quarks are included. Interactions modify the dispersion relation and lead to a nonvanishing matrix element. Quark-quark interactions can be treated either perturbatively in lowest order of the strong coupling constant $\alpha_{s}$

$$
\mu_{i} \simeq p_{F, i}\left(1+\frac{2}{3 \pi} \alpha_{s}\right), \quad \alpha_{s}=\frac{g^{2}}{4 \pi}, \quad i=u, d
$$

or due to the effect of finite quark masses

$$
\mu_{i}=\sqrt{p_{F, i}^{2}+m_{i}^{2}} \simeq p_{F, i}\left[1+\frac{1}{2}\left(\frac{m_{i}}{p_{F, i}}\right)^{2}\right], \quad i=u, d, e .
$$

Using Eqs. C6, (C7) and the $\beta$-equilibrium condition $\mu_{d}=\mu_{u}+\mu_{e}$ one can find the equivalent expressions for the angle $\theta_{d e}$ corresponding to Eq. (C5)

$$
\theta_{d e}^{2} \simeq\left\{\begin{array}{c}
\frac{4}{3 \pi} \alpha_{s} \\
\frac{m_{d}^{2}}{p_{F, e} p_{F, d}}\left[1-\left(\frac{m_{u}}{m_{d}}\right)^{2}\left(\frac{p_{F, d}}{p_{F, u}}\right)-\left(\frac{m_{e}}{m_{d}}\right)^{2}\left(\frac{p_{F, d}}{p_{F, e}}\right)\right],
\end{array}\right.
$$

which is crucial to calculate the emissivity, see also [29].

\section{Appendix D: Contractions}

The contraction of the matrix elements of the leptonic tensor (21) gives

$$
\begin{aligned}
\mathcal{L}^{00}\left(q_{1}, q_{2}\right) & =8\left(q_{1}^{0} q_{2}^{0}+\mathbf{q}_{\mathbf{1}} \cdot \mathbf{q}_{\mathbf{2}}\right) \\
\mathcal{L}^{0 i}\left(q_{1}, q_{2}\right) & =8\left[q_{1}^{0} q_{2}^{i}+q_{1}^{i} q_{2}^{0}-i \epsilon^{i j k} q_{1 j} q_{2 k}\right] \\
\mathcal{L}^{i 0}\left(q_{1}, q_{2}\right) & =8\left[q_{1}^{0} q_{2}^{i}+q_{1}^{i} q_{2}^{0}+i \epsilon^{i j k} q_{1 j} q_{2 k}\right] \\
\mathcal{L}^{i j}\left(q_{1}, q_{2}\right) & =8\left[\delta^{i j}\left(q_{1}^{0} q_{2}^{0}-\mathbf{q}_{\mathbf{1}} \cdot \mathbf{q}_{\mathbf{2}}\right)+q_{1}^{i} q_{2}^{j}+q_{1}^{j} q_{2}^{i}-i \epsilon^{i j k l} q_{1 k} q_{2 l}\right]
\end{aligned}
$$

The elements of the tensors $\mathcal{T}_{\mu \nu}^{ \pm}(\hat{p}, \hat{k}), \widetilde{\mathcal{T}}_{\mu \nu}^{ \pm}(\hat{p}, \hat{k}), \mathcal{W}_{\mu \nu}^{ \pm}(\hat{p}, \hat{k}), \widetilde{\mathcal{W}}_{\mu \nu}^{ \pm}(\hat{p}, \hat{k})$ in Eq. B4 become

$$
\begin{array}{ll}
\mathcal{T}_{00}^{ \pm}(\hat{p}, \hat{k})=1+\hat{p} \cdot \hat{k}+\hat{m}_{u} \hat{m}_{d}, & \widetilde{\mathcal{T}}_{00}^{ \pm}(\hat{p}, \hat{k})=1+\hat{p} \cdot \hat{k}-\hat{m}_{u} \hat{m}_{d} \\
\mathcal{T}_{0 i}^{ \pm}(\hat{p}, \hat{k})= \pm\left(\hat{p}_{i}+\hat{k}_{i}\right), & \widetilde{\mathcal{T}}_{0 i}^{ \pm}(\hat{p}, \hat{k})= \pm\left(\hat{p}_{i}+\hat{k}_{i}\right) \\
\mathcal{T}_{i 0}^{ \pm}(\hat{p}, \hat{k})= \pm\left(\hat{p}_{i}+\hat{k}_{i}\right), & \widetilde{\mathcal{T}}_{i 0}^{ \pm}(\hat{p}, \hat{k})= \pm\left(\hat{p}_{i}+\hat{k}_{i}\right) \\
\mathcal{T}_{i j}^{ \pm}(\hat{p}, \hat{k})=\delta_{i j}\left(1-\hat{p} \cdot \hat{k}-\hat{m}_{u} \hat{m}_{d}\right)+\hat{p}_{i} \hat{k}_{j}+\hat{k}_{i} \hat{p}_{j}, & \widetilde{\mathcal{T}}_{i j}^{ \pm}(\hat{p}, \hat{k})=\delta_{i j}\left(1-\hat{p} \cdot \hat{k}+\hat{m}_{u} \hat{m}_{d}\right)+\hat{p}_{i} \hat{k}_{j}+\hat{k}_{i} \hat{p}_{j}
\end{array}
$$




$$
\begin{aligned}
& \mathcal{W}_{00}^{ \pm}(\hat{p}, \hat{k})=\widetilde{\mathcal{W}}_{00}^{ \pm}(\hat{p}, \hat{k})=0, \\
& \mathcal{W}_{0 i}^{ \pm}(\hat{p}, \hat{k})=\widetilde{\mathcal{W}}_{0 i}^{ \pm}(\hat{p}, \hat{k})=-i \epsilon_{i j k} \hat{p}^{j} \hat{k}^{k} \\
& \mathcal{W}_{i 0}^{ \pm}(\hat{p}, \hat{k})=\widetilde{\mathcal{W}}_{i 0}^{ \pm}(\hat{p}, \hat{k})=+i \epsilon_{i j k} \hat{p}^{j} \hat{k}^{k} \\
& \mathcal{W}_{i j}^{ \pm}(\hat{p}, \hat{k})=\widetilde{\mathcal{W}}_{i j}^{ \pm}(\hat{p}, \hat{k})=\mp i \epsilon_{i j k}\left(\hat{p}^{k}-\hat{k}^{k}\right)+i \epsilon_{i j k l} \hat{p}^{k} \hat{k}^{l}
\end{aligned}
$$

With (D1), (D2) and (D3) we obtain for the Lorentz contraction

$$
\begin{aligned}
\mathcal{L}^{\mu \nu}\left(q_{1}, q_{2}\right) \mathcal{T}_{\mu \nu}^{ \pm}(\hat{p}, \hat{k}) & =16\left[\left(q_{1}^{0} \mp \mathbf{q}_{\mathbf{1}} \cdot \hat{p}\right)\left(q_{2}^{0} \mp \mathbf{q}_{\mathbf{2}} \cdot \hat{k}\right)+\left(q_{1}^{0} \mp \mathbf{q}_{\mathbf{1}} \cdot \hat{k}\right)\left(q_{2}^{0} \mp \mathbf{q}_{\mathbf{2}} \cdot \hat{p}\right)-\left(q_{1}^{0} q_{2}^{0}-\mathbf{q}_{\mathbf{1}} \cdot \mathbf{q}_{\mathbf{2}}\right) \hat{m}_{u} \hat{m}_{d}\right], \\
\mathcal{L}^{\mu \nu}\left(q_{1}, q_{2}\right) \widetilde{\mathcal{T}}_{\mu \nu}^{ \pm}(\hat{p}, \hat{k}) & =16\left[\left(q_{1}^{0} \mp \mathbf{q}_{\mathbf{1}} \cdot \hat{p}\right)\left(q_{2}^{0} \mp \mathbf{q}_{\mathbf{2}} \cdot \hat{k}\right)+\left(q_{1}^{0} \mp \mathbf{q}_{\mathbf{1}} \cdot \hat{k}\right)\left(q_{2}^{0} \mp \mathbf{q}_{\mathbf{2}} \cdot \hat{p}\right)+\left(q_{1}^{0} q_{2}^{0}-\mathbf{q}_{\mathbf{1}} \cdot \mathbf{q}_{\mathbf{2}}\right) \hat{m}_{u} \hat{m}_{d}\right], \\
\mathcal{L}^{\mu \nu}\left(q_{1}, q_{2}\right) \mathcal{W}_{\mu \nu}^{ \pm}(\hat{p}, \hat{k}) & =16\left[\left(q_{1}^{0} \mp \mathbf{q}_{\mathbf{1}} \cdot \hat{k}\right)\left(q_{2}^{0} \mp \mathbf{q}_{\mathbf{2}} \cdot \hat{p}\right)-\left(q_{1}^{0} \mp \mathbf{q}_{\mathbf{1}} \cdot \hat{p}\right)\left(q_{2}^{0} \mp \mathbf{q}_{\mathbf{2}} \cdot \hat{k}\right)\right] .
\end{aligned}
$$

Contraction between leptonic $\mathcal{L}^{\mu \nu}\left(q_{1}, q_{2}\right)$ and hadronic tensor of the normal phase $\mathcal{H}_{\mu \nu}^{(\mathrm{n})}(\hat{p}, \hat{k})$ results in

$$
\begin{aligned}
\mathcal{L}^{\mu \nu}\left(q_{1}, q_{2}\right) \mathcal{H}_{\mu \nu}^{(\mathrm{n})}(\hat{p}, \hat{k})= & \mathcal{L}^{\mu \nu}\left(q_{1}, q_{2}\right) \mathcal{T}_{\mu \nu}^{+}(\hat{p}, \hat{k})+\mathcal{L}^{\mu \nu}\left(q_{1}, q_{2}\right) \widetilde{\mathcal{T}}_{\mu \nu}^{+}(\hat{p}, \hat{k})-\mathcal{L}^{\mu \nu}\left(q_{1}, q_{2}\right) \mathcal{W}_{\mu \nu}^{+} \\
= & 16\left[\left(q_{1}^{0}-\mathbf{q}_{\mathbf{1}} \cdot \hat{p}\right)\left(q_{2}^{0}-\mathbf{q}_{\mathbf{2}} \cdot \hat{k}\right)+\left(q_{1}^{0}-\mathbf{q}_{\mathbf{1}} \cdot \hat{k}\right)\left(q_{2}^{0}-\mathbf{q}_{\mathbf{2}} \cdot \hat{p}\right)\right. \\
& -\left(q_{1}^{0} q_{2}^{0}-\mathbf{q}_{\mathbf{1}} \cdot \mathbf{q}_{\mathbf{2}}\right) m_{u} \hat{m}_{d} \\
& +\left(q_{1}^{0}-\mathbf{q}_{\mathbf{1}} \cdot \hat{p}\right)\left(q_{2}^{0}-\mathbf{q}_{\mathbf{2}} \cdot \hat{k}\right)+\left(q_{1}^{0}-\mathbf{q}_{\mathbf{1}} \cdot \hat{k}\right)\left(q_{2}^{0}-\mathbf{q}_{\mathbf{2}} \cdot \hat{p}\right) \\
& +\left(q_{1}^{0} q_{2}^{0}-\mathbf{q}_{\mathbf{1}} \cdot \mathbf{q}_{\mathbf{2}}\right) \hat{m}_{u} \hat{m}_{d} \\
& \left.-2\left(q_{1}^{0}-\mathbf{q}_{\mathbf{1}} \cdot \hat{k}\right)\left(q_{2}^{0}-\mathbf{q}_{\mathbf{2}} \cdot \hat{p}\right)+2\left(q_{1}^{0}-\mathbf{q}_{\mathbf{1}} \cdot \hat{p}\right)\left(q_{2}^{0}-\mathbf{q}_{\mathbf{2}} \cdot \hat{k}\right)\right] \\
= & 64\left(q_{1}^{0}-\mathbf{q}_{\mathbf{1}} \cdot \hat{p}\right)\left(q_{2}^{0}-\mathbf{q}_{\mathbf{2}} \cdot \hat{k}\right) \\
= & 64 q_{1}^{0} q_{2}^{0}\left(1-\hat{q}_{1} \cdot \hat{p}\right)\left(1-\hat{q}_{2} \cdot \hat{k}\right) .
\end{aligned}
$$

Contraction between the leptonic $\mathcal{L}^{\mu \nu}\left(q_{1}, q_{2}\right)$ and the hadronic tensor of the anomalous phase $\mathcal{H}_{\mu \nu}^{(a)}(\hat{p}, \hat{k})$ leads to

$$
\begin{aligned}
\mathcal{L}^{\mu \nu}\left(q_{1}, q_{2}\right) \mathcal{H}_{\mu \nu}^{(\mathrm{a})}(\hat{p}, \hat{k})= & \mathcal{L}^{\mu \nu}\left(q_{1}, q_{2}\right) \mathcal{T}_{\mu \nu}^{-}(\hat{p}, \hat{k})+\mathcal{L}^{\mu \nu}\left(q_{1}, q_{2}\right) \mathcal{T}_{\mu \nu}^{+}(\hat{p}, \hat{k}) \\
& +\mathcal{L}^{\mu \nu}\left(q_{1}, q_{2}\right) \widetilde{\mathcal{T}}_{\mu \nu}^{-}(\hat{p}, \hat{k})+\mathcal{L}^{\mu \nu}\left(q_{1}, q_{2}\right) \widetilde{\mathcal{T}}_{\mu \nu}^{+}(\hat{p}, \hat{k}) \\
& -2\left[\mathcal{L}^{\mu \nu}\left(q_{1}, q_{2}\right) \mathcal{W}_{\mu \nu}^{+}(\hat{p}, \hat{k})-\mathcal{L}^{\mu \nu}\left(q_{1}, q_{2}\right) \mathcal{W}_{\mu \nu}^{-}(\hat{p}, \hat{k})\right] \\
= & 64\left[\left(q_{1}^{0}+\mathbf{q}_{\mathbf{1}} \cdot \hat{k}\right)\left(q_{2}^{0}+\mathbf{q}_{\mathbf{2}} \cdot \hat{p}\right)+\left(q_{1}^{0}-\mathbf{q}_{\mathbf{1}} \cdot \hat{p}\right)\left(q_{2}^{0}-\mathbf{q}_{\mathbf{2}} \cdot \hat{k}\right)\right] \\
= & 64 q_{1}^{0} q_{2}^{0}\left[\left(1+\hat{q}_{1} \cdot \hat{k}\right)\left(1+\hat{q}_{2} \cdot \hat{p}\right)+\left(1-\hat{q}_{1} \cdot \hat{p}\right)\left(1-\hat{q}_{2} \cdot \hat{k}\right)\right] .
\end{aligned}
$$

[1] T. Klähn et al., Phys. Rev. C 74, 035802 (2006).

[2] D. Blaschke, J. Schaffner-Bielich and H.-J. Schulze (eds.), Topical Issue on "Exotic Matter in Neutron Stars", Eur. Phys. J. A, 52, 71 (2016).

[3] P. Demorest, T. Pennucci, S. Ransom, M. Roberts and J. Hessels, Nature 467, 1081 (2010).

[4] E. Fonseca, T. T. Pennucci, J. A. Ellis, I. H. Stairs, D. J. Nice, et al., arXiv:1603.00545 [astro-ph.HE].

[5] J. Antoniadis, P. C. C. Freire, N. Wex, T. .M. Tauris, R. S. Lynch, et al., Science 340, 448 (2013).

[6] S. Bogdanov, Astrophys. J. 762, 96 (2013).

[7] F. Özel, Nature 441, 1115 (2006).

[8] M. Alford, D. Blaschke, A. Drago, T. Klähn, G. Pagliara and J. Schaffner-Bielich, Nature 445, E7 (2007).

[9] T. Klähn, D. Blaschke, F. Sandin, C. Fuchs, A. Faessler, H. Grigorian, G. Röpke and J. Trümper, Phys. Lett. B 654, 170 (2007).

[10] D. B. Blaschke, D. Gomez Dumm, A. G. Grunfeld, T. Klähn and N. N. Scoccola, Phys. Rev. C 75, 065804 (2007).

[11] A. G. Grunfeld, J. Berdermann, D. B. Blaschke, D. Gomez Dumm, T. Klähn and N. N. Scoccola, Int. J. Mod. Phys. E 16, 2842 (2007).

[12] M. G. Alford, G. F. Burgio, S. Han, G. Taranto and D. Zappalá, Phys. Rev. D 92, no. 8, 083002 (2015).

[13] M. Alford, M. Braby, M. W. Paris and S. Reddy, Astro- 
phys. J. 629, 969 (2005).

[14] D. Blaschke, D. E. Alvarez-Castillo and S. Benic, PoS CPOD 2013, 063 (2013).

[15] S. Benic, D. Blaschke, D. E. Alvarez-Castillo, T. Fischer and S. Typel, Astron. Astrophys. 577, A40 (2015).

[16] https://heasarc.gsfc.nasa.gov/docs/nicer

[17] J. A. Pons, S. Reddy, M. Prakash, J. M. Lattimer and J. A. Miralles, Astrophys. J. 513, 780 (1999).

[18] T. Fischer, S. C. Whitehouse, A. Mezzacappa, F.K. Thielemann and M. Liebendörfer, Astron. Astrophys. 517, A80 (2010).

[19] L. Hüdepohl, B. Müller, H.-T. Janka, A. Marek and G. G. Raffelt, Phys. Rev. Lett. 104, 251101 (2010); Erratum: [Phys. Rev. Lett. 105, 249901 (2010)].

[20] L. Roberts, S. Reddy and G. Shen, Phys. Rev. C 86, 065803 (2012).

[21] G. Martinez-Pinedo, T. Fischer, A. Lohs and L. Huther, Phys. Rev. Lett. 109, 251104 (2012).

[22] T. Fischer, Astron. Astrophys. (in press) (2016).

[23] J. A. Pons, A. W. Steiner, M. Prakash and J. M. Lattimer, Phys. Rev. Lett. 86, 5223 (2001).

[24] K. Nakazato, K. Sumiyoshi and S. Yamada, Phys. Rev. D 77, 103006 (2008).

[25] I. Sagert, T. Fischer, M. Hempel, G. Pagliara, J. Schaffner-Bielich, et al., Phys. Rev. Lett. 102, 081101 (2009).

[26] T. Fischer, I. Sagert, G. Pagliara, M. Hempel, J. Schaffner-Bielich, et al., Astrophs. J. Suppl. 194, 39 (2011).

[27] B. Dasgupta, T. Fischer, S. Horiuchiu, M. Liebendörfer, A. Mirizzi, et al., Phys. Rev. D 81, 103005 (2010).

[28] N. Iwamoto, Phys. Rev. Lett. 44, 1637 (1980).

[29] N. Iwamoto, Annals Phys. 141, 1 (1982).

[30] P. Haensel, J. L. Zdunik and R. Schaeffer, Astron. Astrophys. 160, 121 (1986).

[31] A. Burrows, Phys. Rev. Lett. 44, 1640 (1980).

[32] N. Iwamoto, Phys. Rev. D 28, 2353 (1983).

[33] W. C. G. Ho and C. O. Heinke, Nature 462, 71 (2009).

[34] W. C. G. Ho, K. G. Elshamouty, C. O. Heinke and A. Y. Potekhin, Phys. Rev. C 91, no. 1, 015806 (2015).

[35] H. Grigorian, D. N. Voskresensky and D. Blaschke, Eur. Phys. J. A 52, no. 3, 67 (2016).

[36] S. B. Ruester, V. Werth, M. Buballa, I. A. Shovkovy and D. H. Rischke, Phys. Rev. D 72, 034004 (2005).

[37] D. Blaschke, S. Fredriksson, H. Grigorian, A. M. Öztas and F. Sandin, Phys. Rev. D 72, 065020 (2005).

[38] M. Buballa, Phys. Rept. 407, 205 (2005).

[39] J. Madsen, Phys. Rev. Lett. 85, no. 1, 10 (2000).

[40] D. Blaschke, T. Klähn and D. N. Voskresensky, Astrophys. J. 533, 406 (2000).

[41] S. Borsanyi, Z. Fodor, C. Hoelbling, S. D. Katz, S. Krieg and K. K. Szabo, Phys. Lett. B 730, 99 (2014).

[42] A. Bazavov et al. [HotQCD Collaboration], Phys. Rev. D 90, 094503 (2014).
[43] D. B. Blaschke, F. Sandin, V. V. Skokov and S. Typel, Acta Phys. Polon. Supp. 3, 741 (2010).

[44] A. Ayriyan, J. Berdermann, D. Blaschke and R. Lastowiecki, arXiv:1608.07875 [hep-ph].

[45] H. Grigorian, D. Blaschke and D. Voskresensky, Phys. Rev. C 71, 045801 (2005).

[46] A. Sedrakian, Eur. Phys. J. A 52, 44 (2016).

[47] A. Sedrakian, Astron. Astrophys. 555, L10 (2013).

[48] M. G. Alford, H. Nishimura and A. Sedrakian, Phys. Rev. C 90, no. 5, 055205 (2014).

[49] D. Hess and A. Sedrakian, Phys. Rev. D 84, 063015 (2011).

[50] M. G. Alford, A. Schmitt, K. Rajagopal and T. Schäfer, Rev. Mod. Phys. 80, 1455 (2008).

[51] R. Anglani, R. Casalbuoni, M. Ciminale, N. Ippolito, R. Gatto, M. Mannarelli and M. Ruggieri, Rev. Mod. Phys. 86, 509 (2014).

[52] D. N. Aguilera, D. Blaschke, M. Buballa and V. L. Yudichev, Phys. Rev. D 72, 034008 (2005).

[53] D. N. Aguilera, D. Blaschke, H. Grigorian and N. N. Scoccola, Phys. Rev. D 74, 114005 (2006).

[54] A. Schmitt, Phys. Rev. D 71, 054016 (2005).

[55] A. Schmitt, I. A. Shovkovy and Q. Wang, Phys. Rev. D 73, 034012 (2006).

[56] S. Popov, H. Grigorian and D. Blaschke, Phys. Rev. C 74, 025803 (2006).

[57] D. Blaschke and H. Grigorian, Prog. Part. Nucl. Phys. 59, 139 (2007).

[58] D. B. Blaschke and J. Berdermann, AIP Conf. Proc. 964, 290 (2007).

[59] J. I. Kapusta and C. Gale, Finite-temperature field theory: Principles and applications,' (Cambridge University Press, Cambridge, 2005)

[60] S. B. Ruester, nucl-th/0612090.

[61] M. Abramowitz and I. Stegun, Pocketbook of mathematical functions (Verlag Harri Deutsch, 1984).

[62] H. Grigorian, Phys. Part. Nucl. Lett. 4, 223 (2007).

[63] P. Jaikumar, C. D. Roberts and A. Sedrakian, Phys. Rev. C 73, 042801 (2006).

[64] Q. Wang, Z. G. Wang and J. Wu, Phys. Rev. D 74, 014021 (2006).

[65] B. A. Sa'd, I. A. Shovkovy and D. H. Rischke, Phys. Rev. D 75, 065016 (2007).

[66] L.P. Kadanoff and G. Baym, Quantum Statistical Mechanics (Benjamin,New York,1962).

[67] N. Andersson, Astrophys. J. 502, 708 (1998).

[68] N. Andersson and K. D. Kokkotas, Int. J. Mod. Phys. D 10, 381 (2001).

[69] A. Drago, G. Pagliara and I. Parenti, Astrophys. J. 678, L117 (2008).

[70] M. G. Alford and A. Schmitt, J. Phys. G 34, 67 (2007).

[71] X. Wang, H. Malekzadeh and I. A. Shovkovy, Phys. Rev. D 81, 045021 (2010). 